\title{
Star cluster classification in the PHANGS-HST survey: Comparison between human and machine learning approaches
}

Bradley C. Whitmore, ${ }^{1 \star}$ Janice C. Lee ${ }^{\circledR},{ }^{2,3 \star}$ Rupali Chandar, ${ }^{4}$ David A. Thilker ${ }^{\circledR}, 5$ Stephen Hannon ${ }^{\circledR}, 6$ Wei Wei, ${ }^{7}$ E. A. Huerta, ${ }^{7}$ Frank Bigiel, ${ }^{8}$ Médéric Boquien, ${ }^{9}$ Mélanie Chevance ${ }^{\circledR},{ }^{10}$ Daniel A. Dale, ${ }^{11}$ Sinan Deger, ${ }^{12}$ Kathryn Grasha ${ }^{\circledR},{ }^{13}$ Ralf S. Klessen ${ }^{\circledR},{ }^{14,15}$ J. M. Diederik Kruijssen ${ }^{\circledR},{ }^{10}$ Kirsten L. Larson, ${ }^{3}$ Angus Mok ${ }^{4}$ Erik Rosolowsky ${ }^{\circledR},{ }^{16}$ Eva Schinnerer, ${ }^{17}$ Andreas Schruba, ${ }^{17}$ Leonardo Ubeda, ${ }^{1}$ Schuyler D. Van Dyk ${ }^{\circledR},{ }^{3}$ Elizabeth Watkins ${ }^{10}$ and Thomas Williams ${ }^{(17} 17$

${ }^{1}$ Space Telescope Science Institute, 3700 San Martin Drive, Baltimore, MD 21218, USA

${ }^{2}$ Gemini Observatory/NSF's NOIRLab, 950 N. Cherry Ave, Tucson, AZ 85719, USA

${ }^{3}$ Caltech/IPAC, 1200 California Blvd, Pasadena, CA 91125, USA

${ }^{4}$ Department of Physics, Astronomy, University of Toledo, Toledo, OH 43606, USA

${ }^{5}$ Department of Physics and Astronomy, The Johns Hopkins University, Baltimore, MD 21218, USA

${ }^{6}$ Department of Physics and Astronomy, University of California, Riverside, CA 92521, USA

${ }^{7}$ NCSA, Center for Artificial Intelligence Innovation, University of Illinois at Urbana-Champaign, Urbana, IL 61801, USA

${ }^{8}$ Argelander-Institut für Astronomie, Universität Bonn, Auf dem Hügel 71, D-53121 Bonn, Germany

${ }^{9}$ Centro de Astronomía, (CITEVA), Universidad de Antofagasta, Avenida Angamos 601, Antofagasta 1270300, Chile

${ }^{10}$ Astronomisches Rechen-Institut, Zentrum für Astronomie der Universität Heidelberg, Mönchhofstraße 12-14, D-69120 Heidelberg, Germany

${ }^{11}$ Department of Physics and Astronomy, University of Wyoming, Laramie, WY 82071, USA

${ }^{12}$ TAPIR, California Institute of Technology, Pasadena, CA 91125, USA

${ }^{13}$ Research School of Astronomy and Astrophysics, Australian National University, Canberra, ACT 2611, Australia

${ }^{14}$ Universität Heidelberg, Zentrum für Astronomie, Institut für Theoretische Astrophysik, D-69120 Heidelberg, Germany

${ }^{15}$ Universität Heidelberg, Interdisziplinäres Zentrum für Wissenschaftliches Rechnen, D-69120 Heidelberg, Germany

${ }^{16}$ Department of Physics, University of Alberta, Edmonton, AB T6G 2E1, Canada

${ }^{17}$ Max Planck Institut für Astronomie, Königstuhl 17, D-69117 Heidelberg, Germany

Accepted 2021 July 16. Received 2021 July 16; in original form 2021 March 16

\begin{abstract}
When completed, the PHANGS-HST project will provide a census of roughly 50000 compact star clusters and associations, as well as human morphological classifications for roughly 20000 of those objects. These large numbers motivated the development of a more objective and repeatable method to help perform source classifications. In this paper, we consider the results for five PHANGS-HST galaxies (NGC 628, NGC 1433, NGC 1566, NGC 3351, NGC 3627) using classifications from two convolutional neural network architectures (RESNET and VGG) trained using deep transfer learning techniques. The results are compared to classifications performed by humans. The primary result is that the neural network classifications are comparable in quality to the human classifications with typical agreement around 70 to 80 per cent for Class 1 clusters (symmetric, centrally concentrated) and 40 to 70 per cent for Class 2 clusters (asymmetric, centrally concentrated). If Class 1 and 2 are considered together the agreement is $82 \pm 3$ per cent. Dependencies on magnitudes, crowding, and background surface brightness are examined. A detailed description of the criteria and methodology used for the human classifications is included along with an examination of systematic differences between PHANGS-HST and LEGUS. The distribution of data points in a colour-colour diagram is used as a 'figure of merit' to further test the relative performances of the different methods. The effects on science results (e.g. determinations of mass and age functions) of using different cluster classification methods are examined and found to be minimal.
\end{abstract}

Key words: catalogues-galaxies: star clusters: general.

\section{INTRODUCTION}

The identification of star clusters in external galaxies is useful for a variety of purposes. Besides providing insight into the transition from giant molecular clouds to stars and star clusters, they are also useful as 'clocks' to time this evolution since they can be effectively

^E-mail: whitmore@stsci.edu (BCW); janice.lee@noirlab.edu (JCL) characterized as single-aged stellar populations. Perhaps the most important time-scale for understanding the evolution of galaxies is the rate at which feedback removes the gas from the region around forming stars, limiting the rate at which the gas is used and stars are formed in the galaxy.

Early efforts to classify star clusters in external galaxies relied on visual inspection of photographic plates of nearby galaxies (e.g. Hodge 1981). This resulted in catalogues with typically a few hundred objects for roughly a dozen galaxies (e.g. M31, M33, M81, 
LMC, SMC). The availability of the Hubble Space Telescope (HST), with roughly a factor of ten improvement in spatial resolution, acted as a major catalyst for the study of extragalactic star clusters (e.g. Holtzman et al. 1992; Meurer et al. 1995; Whitmore et al. 1995). With the 10-fold improvement in spatial resolution came a 1000fold increase in the volume that could be searched. Rather than a few hundred clusters in each galaxy, the accompanying improvement in detection level led to typical numbers of clusters for each galaxy in the thousands.

The HST archives contain a few hundred nearby galaxies with data sets that could support detailed studies of clusters (i.e. with three or more bands to enable age-dating through SED modelling). The increase in the number of clusters that might be classified compared to the earlier pre-HST era is therefore a factor of a few hundred [i.e. (300 galaxies $\times 3000$ clusters $) /(10$ galaxies $\times 300$ clusters $)$ ], with a total number of clusters that could be classified around 1 million. Classification of this many objects represents a limiting constraint for the study of clusters in nearby galaxies, and was the primary reason for development of automated, neural network methods for cluster classifications (Messa et al. 2018; Bialopetravičius; Narbutis \& Vansevičius 2019; Grasha et al. 2019; Wei et al. 2020; Pérez et al. 2021).

Using neural networks not only accelerates the process of classifications, which has been a limiting step in the production of star cluster catalogues, it also improves the consistency of the classifications by reducing both random and systematic errors introduced by the subjective nature of human classifications (i.e. the same person may give different classifications for the same object on different occasions). We thus developed a new automated approach to star cluster classifications using neural networks (Wei et al. 2020) as part of the Physics at High Angular Resolution in Nearby Galaxies with the Hubble Space Telescope project (PHANGS-HST); PI: J. C. Lee, GO-15654) (J. C. Lee et al., in preparation). ${ }^{1}$ PHANGS-HST is a Cycle 26 Treasury program obtaining 5-band UV-optical, F275W (NUV), F336W (U), F438W (B), F555W (V), F814W (I), WFC3 (or ACS in some cases with existing data) imaging for 38 nearby spiral galaxies with previous $\mathrm{CO}(2-1)$ observations from the PHANGSALMA large program (Leroy et al. 2021). ${ }^{2}$

An early attempt to classify clusters using quantitative morphological parameters is described in Whitmore et al. (2014). Using only a simple concentration index $(\mathrm{CI}=$ difference in photometry in circular apertures with radii of 1 and 3 pixel), they were able to work down to $M_{V}=-8$ mag (Vega magnitude system) without degrading the sample with a large fraction of blended stars being misidentified as clusters. This study provided cluster luminosity functions for 20 galaxies, with typically a few hundred clusters in each galaxy.

A citizen science approach to cluster classification was used for the PHAT (Panchromatic Hubble Andromeda Treasury) project (Johnson et al. 2012; Johnson et al. 2015) to accelerate classification. While this works well for nearby well-resolved clusters, exploratory efforts for the more distant galaxies in the Legacy ExtraGalactic Ultraviolet Survey - (Calzetti et al. 2015 - LEGUS) project (out to $\sim 11 \mathrm{Mpc}$ ) were unsuccessful due to the more subtle differences between clusters, associations, stars, and interlopers due to the decreased physical resolution, as reported in Pérez et al. (2021).

Another pioneering study was performed using a machine learning approach developed as part of the LEGUS project. While this was successful for nearby well-resolved clusters (i.e. recovery rates on the order 60 to 70 per cent between human and machine learning

\footnotetext{
${ }^{1}$ https://archive.stsci.edu/hlsp/phangs-hst

${ }^{2} \mathrm{https} / / /$ sites.google.com/view/phangs/home
}

approaches), exploratory efforts for the more distant galaxies, and for the compact associations, showed lower recovery rates. Subsequently, Pérez et al. (2021) developed multiscale convolutional neural network models for the LEGUS project, with agreement fractions on par with the performance of our models in Wei et al. (2020). An additional contribution is Bialopetravičius et al. (2019), who used neural network classifications based on simulations for resolved star clusters in M31.

Many of the most promising recent machine learning approaches make use of neural networks. Given the small sizes of existing, human-labelled HST star cluster samples (approximately $2000 \mathrm{ob}-$ jects per class spread out over 39 fields) relative to the samples needed for the robust training of neural networks, we decided to use deep transfer learning techniques as described in detail in Wei et al. (2020). That is, a neural network model, pre-trained on images of everyday objects from the ImageNet data set (Deng et al. 2009), is fine-tuned using HST image data of clusters with human classifications, rather than training all of the layers in the network from scratch. The results of our proof-of-concept experiment in Wei et al. (2020) were encouraging, as the prediction accuracies based on testing with the first PHANGS-HST galaxy observed, NGC 1559, were found to be competitive with the consistency between different human classifications. In this paper, we take an expanded look at the performance of the neural network models presented in Wei et al. (2020) by applying the models to star clusters in five additional galaxies, and examining the accuracies as functions of various properties including magnitudes, crowding, background surface brightness, and colours.

We expect our neural network models and cluster catalogues to evolve and improve with time. The cluster catalogues used for this paper are from version 0.9 of the PHANGS-HST pipeline (D. Thilker et al., in preparation) and neural network models from Wei et al. (2020). As new data sets and algorithms are produced they will be made available on the PHANGS-HST website at https://archive.stsci.edu/hlsp/phangs-hst, and will be evaluated in future papers from our team.

The paper is organized as follows. In Section 2, we describe the data set and how the machine learning classifications were performed. In Section 3, we describe the methodology for the human cluster classifications and in Section 4, we compare the agreement fraction for human classifications with the machine learning classifications. In Section 5, we develop several 'figures of merit' based on the distribution of data points in the U-B versus V-I colour-colour diagram, in an attempt to determine which classifications provide the best results. Section 6 investigates issues related to completeness while the dependence of various science results on different methods of classifying clusters is examined in Section 7. Section 8 describes plans to improve the machine learning classifications in the future. A summary and conclusions are presented in Section 9. An appendix provides a basic description of the convolutional neural network models described in Wei et al. (2020) and a step-by-step tutorial on using the models to classify cluster candidates with five band HST imaging.

\section{SAMPLE, DATA, AND MACHINE LEARNING CLASS IF I CATION}

In this paper, we use five galaxies from the PHANGS-HST sample (NGC 628, NGC 1433, NGC 1566, NGC 3351, and NGC 3627) to evaluate the agreement fractions between classifications made by humans and the convolutional neural network (i.e. machine learning) models of Wei et al. (2020). These particular galaxies are chosen because cluster catalogues with human classifications have been 
released by the LEGUS program: https://archive.stsci.edu/prepds/l egus/dataproducts-public.html (Adamo et al. 2017), which provide a point of comparison for both the PHANGS-HST human and machine learning classifications.

HST imaging in five bands, F275W (NUV), F336W (U), F438W (B), F555W (V), F814W (I), WFC3 (or ACS in some cases with existing data), were obtained by LEGUS for all five galaxies. PHANGS-HST obtained imaging in an additional WFC3 pointing for two of the galaxies, NGC 3351 and NGC 3627, to complete coverage of the area of disks mapped in $\mathrm{CO}(2-1)$, and to support joint ALMA-HST analysis (J. C. Lee et al., in preparation).

With these data, $V$-band selected catalogues of compact star clusters and associations, which include five-band aperture photometry, human classifications, and ages, masses, and reddenings derived from SED fitting, were produced by both programs. We have performed a cross-match of the catalogues. For this paper, we primarily study the objects in common between the two catalogues and only use the photometry and physical properties resulting from the PHANGS-HST pipeline. This allows our analysis to focus on differences resulting from classification methodology rather than from detection, selection, and basic photometric procedures such as aperture corrections (e.g. see D. Thilker et al., in preparation and S. Deger et al., in preparation for discussion of these properties).

We have incorporated the neural network models of Wei et al. (2020) into the PHANGS-HST pipeline and use them to produce classifications for all of these objects. As mentioned above, a deep transfer learning approach was used to train models with two different architectures, ResNet18 (He et al. 2016) (=RESNET hereafter) and VGG1 9 (Simonyan \& Zisserman 2014) with batch normalization VGG19-BN (=VGG hereafter). Briefly, ResNet18 is a convolutional neural network that is 18 layers deep. This architecture introduced a number of innovations, skip connections, and batch normalization, that enabled the training of very deep neural networks (hundreds of layers). VGG is a convolutional neural network with a depth of 19 layers. Both networks are open source, and thus their weights may be readily adjusted and tuned through transfer learning to be used for a variety of image recognition tasks.

Two different training sets were used in Wei et al. (2020): (1) '3-person consensus' classifications for clusters in 29 LEGUS galaxies (11268 objects), based on the mode of classifications made by three people as published in the LEGUS cluster catalogues, and (2) single-person classifications for 10 LEGUS galaxies (5488 objects) performed by $\mathrm{BCW}$, the first author of this paper, who also is performing the human classifications for the PHANGS-HST project. Models based on the two different training sets have similar performance. Here, we use the models trained using the BCW-only classifications to perform the PHANGS-HST classifications, and examine results from both the RESNET and VGG architectures. Hence, for all objects in common between the PHANGS-HST and LEGUS cluster catalogues, we compare classifications from four different sources: PHANGS-HST human (BCW-only), LEGUS human (3-person consensus for NGC 628, NGC 1433, and NGC 1566; and BCW-only for NGC 3351 and NGC 3627), RESNET and VGG. We note that two of the ten galaxies in the Wei et al. (2020) BCW-only training set are two of the program galaxies in this study, hence the two samples are not completely independent. This is probably why the performance for these two galaxies is roughly 10 per cent better than for the other three, as shown in Table 1 and discussed later in the text.

Human classifications are determined for sources as faint as $m_{V}=$ 22.5 to 24 mag in the Vega magnitude system depending on the number of candidate clusters in the galaxy (i.e. brighter limits are used for the richer galaxies since the numbers to visually examine can become prohibitive, i.e. more than 10000 in a single galaxy). We also determine neural network classifications for sources up to about one magnitude fainter, and examine the performance of the models for these faint sources in this paper.

\section{HUMAN CLASSIFICATION}

Human classification of star clusters during the selection process has been an important step in most studies of extragalactic star cluster systems, as reviewed by Adamo et al. 2020). The methods developed and lessons learned lay the foundation for the development of automated, reliable cluster classification methods.

\subsection{Background and history}

In this section, we describe the approach used for human classification of clusters in the 38 PHANGS-HST galaxies. The methodology and criteria are very similar to those previously applied to the LEGUS cluster candidate catalogues (Adamo et al. 2017), which were used as training sets for the RESNET and VGG neural network models developed by Wei et al. (2020).

Based on experience, we have previously found that roughly 50 per cent of clusters observed in nearby $(<10 \mathrm{Mpc})$ spiral galaxies with $H S T$ can be reliably classified in just a glance; perhaps 25 per cent can be reliably classified after more careful study; and the remaining $\sim 25$ per cent are often challenging, with properties that make it difficult to confidently establish their classification, for example whether a source is a single star or a very compact cluster. This level of classification is sufficient to determine clear correlations between the classes and various physical properties of the cluster populations, such as their colour, mass, and age distributions. This demonstrates the utility of human classifications, even if they are subjective. We will examine our classification accuracy in Section 5 , where we use the location of different classes of clusters in a colourcolour diagram to test the quality of the classifications, and will assess the effect different methods of classification have on the determination of the age and mass distributions in Section 7.

Previous works have used different numbers of people to perform the human classifications, from a single person up to large numbers from a citizen science approach (e.g. the PHAT survey; Johnson et al. 2015). Sampling statistics suggest that a larger number of classifiers should result in more robust results, but this assumes that each human classifier uses similar definitions and internal weighting systems. In practice, most studies to date have used either a single person (e.g. Chandar et al. 2010b; Bastian et al. 2014; Silva-Villa et al. 2014, ...), a few people, or as many as eight people (Johnson et al. 2012). For LEGUS, three different people from a pool of roughly a dozen classified each object for most of the galaxies, as described in Adamo et al. (2017) (see also Pérez et al. 2021, and H. Kim et al., in preparation), while 10 galaxies were classified by just one person, BCW.

While automated, algorithmically based approaches to classification might be considered objective in one sense, since they are repeatable, it is important to keep in mind that they are based on subjectively determined training sets. Hence we cannot characterize them as fully objective. We note that we are pursuing two approaches that would be more objective, as discussed in Section 8.3.

\subsection{Procedure}

As discussed in Wei et al. (2020), most early cluster studies in external galaxies provided a single classification class. An exception was 
Table 1. Agreement fractions for the five program galaxies.

\begin{tabular}{|c|c|c|c|c|c|c|}
\hline Classification comparison & Class 1 & Class 2 & Class 3 & Class 4 & mean & Class $1+2$ \\
\hline \multicolumn{7}{|l|}{ NGC 628 (9.8 Mpc) } \\
\hline PHANGS versus LEGUS & 0.81 & 0.54 & 0.58 & 0.43 & 0.59 & 0.85 \\
\hline PHANGS versus RESNET & 0.79 & 0.40 & 0.60 & 0.56 & 0.59 & 0.84 \\
\hline PHANGS versus VGG & 0.82 & 0.49 & 0.58 & 0.57 & 0.61 & 0.84 \\
\hline LEGUS versus RESNET & 0.78 & 0.38 & 0.49 & 0.54 & 0.55 & 0.81 \\
\hline LEGUS versus VGG & 0.78 & 0.35 & 0.46 & 0.52 & 0.53 & 0.81 \\
\hline mean for above values & $0.80(0.02)^{a}$ & $0.43(0.08)$ & $0.54(0.06)$ & $0.52(0.06)$ & $0.57(0.04)$ & $0.83(0.02)$ \\
\hline RESNET versus VGG & 0.90 & 0.58 & 0.69 & 0.76 & 0.73 & 0.89 \\
\hline \multicolumn{7}{|l|}{ NGC $1433(18.6 \mathrm{Mpc})$} \\
\hline PHANGS versus LEGUS & 0.74 & 0.54 & 0.54 & 0.59 & 0.60 & 0.80 \\
\hline PHANGS versus RESNET & 0.76 & 0.56 & 0.64 & 0.71 & 0.67 & 0.76 \\
\hline PHANGS versus VGG & 0.81 & 0.54 & 0.58 & 0.64 & 0.64 & 0.71 \\
\hline LEGUS versus RESNET & 0.68 & 0.56 & 0.53 & 0.56 & 0.58 & 0.68 \\
\hline LEGUS versus VGG & 0.68 & 0.56 & 0.53 & 0.56 & 0.58 & 0.68 \\
\hline mean for above values & $0.73(0.06)$ & $0.55(0.01)$ & $0.56(0.05)$ & $0.62(0.06)$ & $0.62(0.04)$ & $0.74(0.04)$ \\
\hline RESNET versus VGG & 0.88 & 0.69 & 0.76 & 0.86 & 0.80 & 0.86 \\
\hline \multicolumn{7}{|l|}{ NGC $1566(17.7 \mathrm{Mpc})$} \\
\hline PHANGS versus LEGUS & 0.80 & 0.50 & 0.47 & 0.49 & 0.56 & 0.82 \\
\hline PHANGS versus RESNET & 0.79 & 0.52 & 0.49 & 0.66 & 0.62 & 0.82 \\
\hline PHANGS versus VGG & 0.82 & 0.53 & 0.55 & 0.68 & 0.64 & 0.82 \\
\hline LEGUS versus RESNET & 0.78 & 0.44 & 0.51 & 0.48 & 0.55 & 0.80 \\
\hline LEGUS versus VGG & 0.80 & 0.47 & 0.50 & 0.50 & 0.57 & 0.80 \\
\hline mean for above values & $0.80(.01)$ & $0.49(.04)$ & $.50(0.03)$ & $0.56(0.10)$ & $0.59(0.04)$ & $0.81(0.01)$ \\
\hline RESNET versus VGG & 0.88 & 0.62 & 0.71 & 0.89 & 0.77 & 0.88 \\
\hline \multicolumn{7}{|l|}{ NGC $3351^{b, c}(10.0 \mathrm{Mpc})$} \\
\hline PHANGS versus LEGUS & 0.82 & 0.53 & 0.46 & 0.73 & 0.64 & 0.81 \\
\hline PHANGS versus RESNET & 0.81 & 0.54 & 0.49 & 0.74 & 0.64 & 0.81 \\
\hline PHANGS versus VGG & 0.82 & 0.54 & 0.51 & 0.72 & 0.65 & 0.78 \\
\hline LEGUS versus RESNET & 0.83 & 0.62 & 0.63 & 0.74 & 0.70 & 0.86 \\
\hline LEGUS versus VGG & 0.85 & 0.60 & 0.56 & 0.69 & 0.67 & 0.81 \\
\hline mean for above values & $0.82(0.01)$ & $0.57(0.04)$ & $0.53(0.07)$ & $0.72(0.02)$ & $0.66(0.03)$ & $0.82(0.03)$ \\
\hline RESNET versus VGG & 0.90 & 0.72 & 0.79 & 0.90 & 0.83 & 0.89 \\
\hline \multicolumn{7}{|l|}{ NGC $3627^{b}(11.3 \mathrm{Mpc})$} \\
\hline PHANGS versus LEGUS & 0.82 & 0.68 & 0.63 & 0.73 & 0.72 & 0.89 \\
\hline PHANGS versus RESNET & 0.81 & 0.60 & 0.60 & 0.72 & 0.68 & 0.90 \\
\hline PHANGS versus VGG & 0.82 & 0.63 & 0.66 & 0.72 & 0.71 & 0.90 \\
\hline LEGUS versus RESNET & 0.88 & 0.74 & 0.74 & 0.84 & 0.80 & 0.93 \\
\hline LEGUS versus VGG & 0.86 & 0.72 & 0.76 & 0.83 & 0.79 & 0.92 \\
\hline mean for above values & $0.84(.03)$ & $0.67(.06)$ & $0.68(0.07)$ & $0.77(0.06)$ & $0.74(0.05)$ & $0.91(0.02)$ \\
\hline RESNET versus VGG & 0.91 & 0.75 & 0.80 & 0.84 & 0.82 & 0.94 \\
\hline mean of means $(n=5)$ & $0.80(0.02)$ & $0.54(0.04)$ & $0.56(0.03)$ & $0.64(0.05)$ & $0.64(0.03)$ & $0.82(0.03)$ \\
\hline
\end{tabular}

Notes. Agreement fractions obtained by requiring one-to-one matches in position with a 2 pixel radius (i.e. using the intersection rather than union of the two studies being compared), separately using both studies as the denominator, and taking the mean of the two determinations. ${ }^{a}$ Values in parenthesis are uncertainties in the mean (i.e. the stddev divided by the sqrt of $\mathrm{N}$ ).

${ }^{b}$ Classifications for NGC 3351 and NGC 3627 were done for LEGUS by BCW, and were therefore part of the BCW-only classifications used in the development of the Wei et al. (2020) model. This may have resulted in the slightly better agreement fractions for these two galaxies in this table.

${ }^{c}$ The NGC 3351 LEGUS field of view was roughly 30 per cent smaller than the fields of view for the other studies. Only the overlapping fields of view were used to calculate the agreement fractions.

the study of Schweizer et al. (1996) who defined nine object types and grouped them into two classes: candidate globular clusters and extended stellar associations. Bastian et al. (2012) classified star clusters in M83 as either symmetric or asymmetric clusters, and argued that the difference between the results of their study and Chandar et al. (2010b) (who used both human and automated cluster catalogues) is largely due to the inclusion of asymmetric clusters. Following this work, many studies in the field, including LEGUS (Calzetti et al. 2015), began differentiating candidate clusters into two or three different categories, so that they could be studied separately or together depending on the goals of the project. It appears that this dichotomy, which has been characterized as 'exclusive' (symmetric clusters) and 'inclusive' (symmetric and asymmetric clusters and in some cases also small associations) by Krumholz, McKee \& Bland-Hawthorn (2019), can explain much of the difference in the slopes of the age distributions in different studies, as first suggested by Bastian et al. (2012) (also see Adamo et al. 2020 for a recent review). We address this issue by presenting age distribution results from different combinations of classes (see below) in Section 7. 
In LEGUS, cluster candidates are sorted into four classes based on their morphological appearance as follows (Adamo et al. 2017; Cook et al. 2019)

(i) Class 1: compact, symmetric, single central peak, radial profile extended relative to point source

(ii) Class 2: asymmetric, single central peak, radial profile extended relative to point source

(iii) Class 3: asymmetric, multiple peaks

(iv) Class 4: not a star cluster (image artefacts, background galaxies, pairs and multiple stars in crowded regions, individual stars.)

We adopt the same general classification system for this paper. We refer to Classes 1, 2, and 3 as symmetric clusters, asymmetric clusters, and compact associations, respectively.

Our primary focus is to identify clusters and groups of stars that are likely to have formed together. Class 1 and 2 objects are referred to as 'clusters', simply based on their centrally peaked profiles, and Class 3 objects are referred to as 'associations' because they have multiple peaks. We note that the topic of associations has largely been superseded in the PHANGS-HST project by the study of K. Larson et al. (in preparation), who use a more uniform hierarchical approach to finding multiscale associations.

These morphological classifications do not provide an unambiguous way of assessing whether or not clusters are bound (have negative energy) or unbound (positive energy), but the bound fraction should depend at least somewhat on the class (e.g. Kruijssen et al. 2012; Ginsburg et al. 2016; Grudić et al. 2020). In general, centrally concentrated clusters that have survived for many crossing times (i.e. are older than $10 \mathrm{Myr}$ - see Gieles \& Portegies Zwart 2011) are likely to be gravitationally bound, while the candidates younger than this contain an unknown mix of bound and unbound clusters, where the bound fraction is predicted to increase towards lower classes.

In the youngest $(<10 \mathrm{Myr})$ clusters, individual bright, massive stars can lead to an asymmetric appearance, regardless of the spatial distribution of the more numerous, lower mass stars, or the internal energy state of a cluster. Clusters naturally become smoother in appearance over time as these massive stars die off. In bound clusters, the distribution becomes smoother due to dynamical interactions between the stars, causing them to relax (e.g. Girichidis et al. 2012; Parker \& Meyer 2012; Parker et al. 2014). In unbound cluster candidates, the distribution becomes smoother by ballistic dispersal (e.g. Baumgardt \& Kroupa 2007; Ward \& Kruijssen 2018; Ward, Kruijssen \& Rix 2020; Wright 2020). In general, all clusters lose mass continuously starting shortly after they form. Therefore, the evolution of clusters should naturally result in some correlation between age and class, where we expect a larger fraction of symmetric clusters (Class 1) at old ages rather than at young ages. Future simulations of evolving clusters which include both gas and stars, and which mimic real observations like the ones made here, would be very helpful to establish how well we can assess the internal energy of the youngest clusters based on their morphologies.

The criteria for Class 1 and 2 are essentially identical for PHANGS-HST and LEGUS. For Class 3, PHANGS-HST uses a more specific definition than LEGUS, namely that at least four stars are detected within a five pixel radius. This is to avoid stellar pairs and triplets which are sometimes included by LEGUS as Class 2 and 3 objects, as will be discussed in Section 6.3.3. The primary rationale for eliminating pairs and triplets is that these have a much higher probability of being chance superpositions in crowded regions than groups/clusters of stars that formed together. Examples of objects in each of the four classes are shown in Fig. 1.

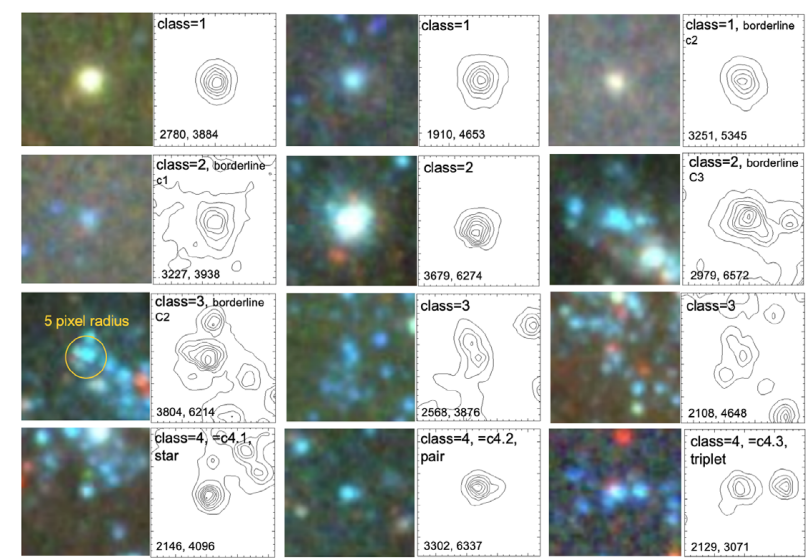

Figure 1. Illustration of the four cluster classification types from the galaxy NGC 628. The colour images (using F814W, F555W, and F336W) show a 40 by 40 pixel field while the contour plots show a 20 by 20 pixel field from the F555W image. Coordinates in the reference frame of the PHANGS-HST images available at https://archive.stsci.edu/hlsp/phangs-hst are included in the bottom left-hand corner of the contour plots. An example of a circle with a 10 pixel diameter (19 pc at the distance of NGC 628) is include for one object to show the scale. Several borderline cases between two classes are included.

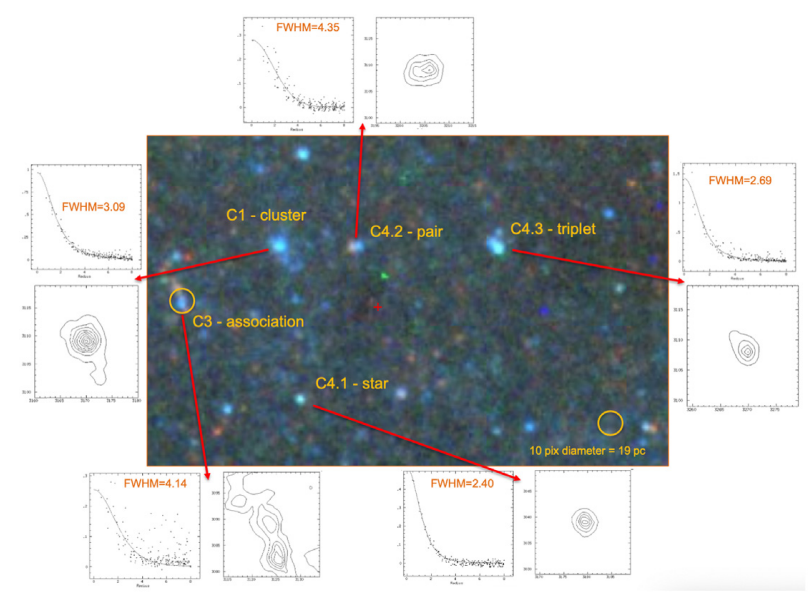

Figure 2. Colour image of a field in NGC 628 illustrating profiles, contour plots, and appearances for five objects. A circle with a diameter of 10 pixels $=$ $19 \mathrm{pc}$ is shown to provide scale. Note that using the colour image helps distinguish pairs (i.e. $\mathrm{C} 4.2$ = pair) when the stars have different colours.

Another difference from LEGUS is that Class 4 (artefacts) has been broken up into 12 subclasses, including $\mathrm{C} 4.1=$ single star, $\mathrm{C} 4.2$ = pair, $\mathrm{C} 4.3=$ triplet, $\mathrm{C} 4.4=$ saturated star, $\ldots$ to $\mathrm{C} 4.12=$ bad pixels. The full set of subclasses are listed in Table 3. One of the reasons for this approach was to be able to test whether machine learning could be better trained if the artefacts were divided into more similar morphologies (see Section 8.1). Another reason was to allow more flexibility if some users wanted to include some objects into Class 3 (e.g. C4.3 = triplets), or wanted to examine the properties of a specific subsample (e.g. C4.6 nucleus of the program galaxy or C4.7 = background galaxies). In most of what follows, the 12 subclasses will be rolled into a single Class 4 , to provide more direct comparisons with LEGUS.

In Fig. 2, we examine a colour image for five objects. Radial profiles and contour plots for various objects are also included. Note that the colour image is especially useful for identifying pairs and triplets when the stars have different colours. The division between 

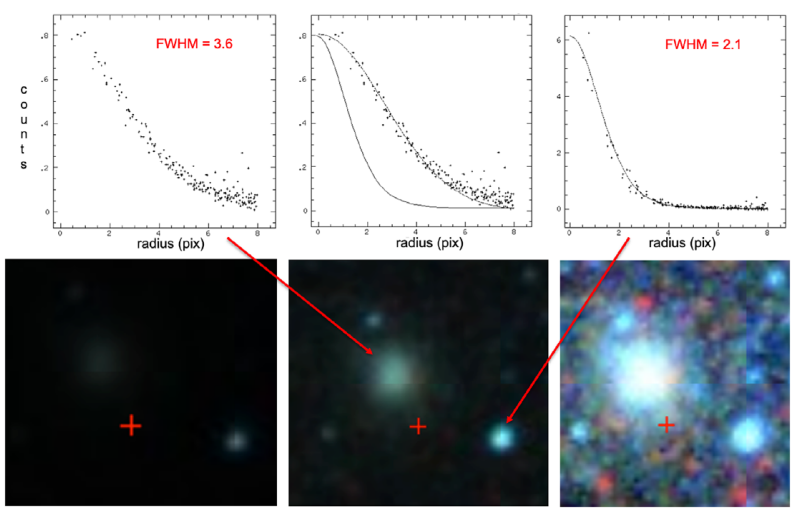

Figure 3. Figure showing how a diffuse Class 1 cluster and nearby star are easily distinguished using different contrast levels in the DS9 display tool (bottom panels - Step 2 in the classification procedure) and the spatial profile using the IMEXAMINE task in IRAF (top panels - Step 3 in the procedure). The upper central panel shows both the cluster and stellar profiles for comparison, with FWHM values of 3.6 pixels (cluster) and 2.1 (star) for these particular objects. The dividing line between stars and clusters is generally around $\mathrm{FWHM}=2.4$ pixels for uncrowded objects with small scatter in the profile.

Class 2 and 3 is sometimes difficult to make. The primary rule is that Class 2 should be centrally concentrated and relatively circular in the contour profiles (i.e. not several objects in a line). The stars in Class 3 objects can generally be seen as separate objects, but in some cases are only visible as strong spurs on the contours.

Distinguishing a bright, well-resolved, isolated star cluster from a single star is generally an easy task for our sample of PHANGS$H S T$ galaxies, as illustrated in Fig. 3. Using a combination of image examination, contrast control, and surface brightness profile review, agreement fractions of over 90 per cent can be obtained between human classifiers, as will be demonstrated in Section 4 .

Of course, it is not always this easy. In most cases, the candidate clusters are fainter, less clearly resolved, and in crowded or high background regions. Below we describe in detail how the human classifications have been done for the PHANGS-HST galaxies.

(i) STEP 1 - IDENTIFY AN INITIAL SAMPLE OF CLUSTER CANDIDATES: A subset of likely clusters are automatically identified in the $\mathrm{F} 555 \mathrm{~W}(V)$ filter image, using the multiple concentration index approach (MCI - see D. Thilker et al., in preparation). Very briefly, the MCI approach uses a combination of photometric measurements with seven apertures ranging from 1 to 5 pixels in radius to distinguish resolved clusters from unresolved stars. The more standard CI-based approach relies on just two apertures (e.g. 1 and 3 pixels; Whitmore et al. 1999; Adamo et al. 2017). The MCI approach typically reduces the source sample from a few hundred thousand point-like objects (mainly stars or close blends) to a few hundred or few thousand cluster candidates in each galaxy. The F555W filter has been selected as a compromise between bluer (e.g. F275W) and redder (e.g. F814W) filters, since both young and old clusters are generally reasonably bright in this filter. We note that all five filters are actually used in the machine learning determinations (Wei et al. 2020).

(ii) STEP 2 - VISUALLY INSPECT IMAGES OF CANDIDATE CLUSTERS: Candidate clusters selected in step 1 are examined using SAOIMAGE DS9, zoomed in by a factor of 2 . In many cases a mere glance at the image will reveal whether the object is a cluster (fuzzy, soft edges) or a star (sharp edges). If not, the contrast is adjusted to compare the candidate cluster with nearby stars of roughly the same brightness. As illustrated in Fig. 3, a star of comparable

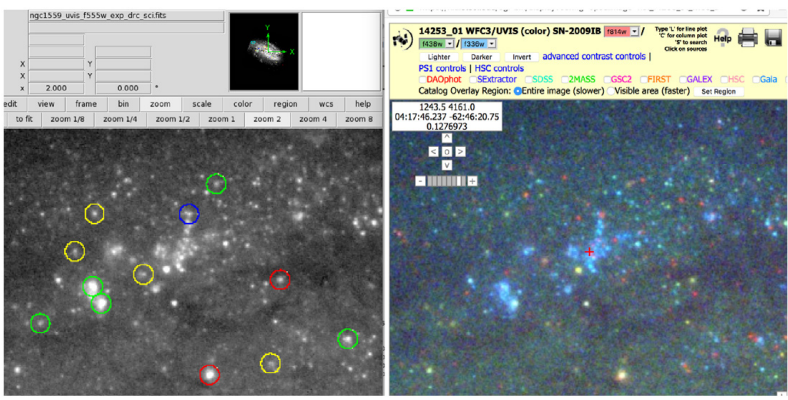

Figure 4. Figure showing how the side-by-side SAOIMAGE DS9 F555W image (left-hand panel) and HLA colour image (right-hand panel) are used to make the classifications; Class $1=$ red, Class $2=$ green, Class $3=$ blue, and Class $4=$ yellow.

luminosity will show up first because of its bright core. As the contrast is increased, the cluster will grow more rapidly and eventually will be larger than the star because of its flatter profile. Even in cases where subsequent tests are not definitive (e.g. determining the FWHM for pairs), this contrast test generally works fairly well.

(iii) STEP 3 - MEASUREMENT OF FWHM AND EXAMINATION OF CONTOURS: The IRAF task IMEXAMINE is used to measure the FWHM of the cluster candidate. Stars typically have FWHM in the range 1.8 to 2.4 pixels in the PHANGS-HST data, while clusters have values in the range 2.4 to 5 (or more) pixels, depending somewhat on the distance of the host galaxy. These correspond to radii for clusters from a few to about $20 \mathrm{pc}$. If the candidate is fairly spherical, and is in an uncrowded region, the scatter around the best-fitting profile is small and the classification as a 'cluster' is fairly secure (e.g. see Fig. 2). However, if the object is elongated, or is in a crowded region, the scatter can be large and this particular test does not help with classification. For example, a close pair of stars can have a large FWHM and a flat looking profile, but the large scatter indicates that such a source is not a cluster (e.g. objects labelled C3 - association, C4.2 - pair, and C4.3 triplet objects in Fig. 2). Contour plots are then used to help determine if a cluster is symmetric (Class 1) or asymmetric (Class 2), and can also help identify stellar pairs (or triplets) and elongated clusters.

Fig. 2 shows several illustrative examples of different contour plots. While the pair (C4.2) in Fig. 2 is obvious, primarily because one star is red and one is blue in the colour image, closer pairs (and triplets) can be challenging to differentiate from slightly extended clusters. In questionable cases, it is often useful to go back and try the contrast test (e.g. does an object grow like two stars, or like an extended cluster).

(iv) STEP 4 - SOURCE MORPHOLOGY IN COLOUR IMAGE: A colour image of each cluster candidate is examined concurrently with steps 2 and 3 . This image is produced by the Hubble Legacy Archive software and is displayed in its interactive display tool https://archive.stsci.edu/hlsp/phangs-hst (HLA - Whitmore et al. 2016). The HLA software has been incorporated into the PHANGS project by coauthor $\mathrm{R}$. White. The colour image is generally created using images in the F336W, F555W, and F814W filters, if available, although in certain cases substituting a different filter (e.g. using the F435W rather than the F336W filter) provides better contrast.

Fig. 4 shows an example of using the DS9 image along side the colour image when making human classifications. The resulting classifications are colour coded: Class $1=$ red, Class $2=$ green, Class $3=$ blue, and Class $4=$ yellow. 


\section{COMPARISON OF RESULTS FROM DIFFERENT CLASSIFICATION METHODS}

As described in Wei et al. (2020), two different deep transfer algorithms (RESNET and VGG) were used to train and test machine learning classifications against both the LEGUS (39 fields in 32 galaxies) and one PHANGS-HST (NGC 1559) human classifications. As briefly summarized in the Introduction, they found prediction accuracies for Classes 1, 2, 3, 4 at roughly the 70, 40, 45, 60 per cent level, respectively. This level of agreement is similar to that found between human-versus-human classification in NGC 4656 (see Wei et al. 2020 for details).

In this section, we quantify how source luminosity, crowding, and background affects the agreement between different classification methods. As discussed in Section 2, the models trained using the BCW-only classifications (i.e. table 1 from Wei et al. 2020) were used to perform the RESNET and VGG classifications.

A few words are in order concerning how we calculate 'agreement fractions'. The first step is to match the two catalogues being compared, so that we are working with the intersection of the two studies rather than the union. A matching radius of two pixels is used. We determine the fraction of exact matches for each class. For that we calculate the ratio of number of matched objects divided by (i) the total number of objects from one study and (ii) the total number of objects in the other study. We adopt the mean of these two ratios as the agreement fraction. We also note that the LEGUS field of view in NGC 3351 is roughly 30 per cent smaller than the PHANGS$H S T$ field of view. Only the region of overlap has been used in the calculation of the agreement fraction and the colour-colour statistics discussed in Section 5.

\subsection{Agreement as a function of cluster class}

We begin by comparing agreement fractions in the galaxy NGC 3351, as shown in Fig. 5. The results for the other galaxies are similar, and are included in Table 1. The comparison between human classifications, made as part of the PHANGS-HST and the LEGUS studies, will be used as the human-to-human baseline in this figure. In both cases, sources were classified by the same person, co-author $\mathrm{BCW}$, but several years apart. The agreement fractions for Classes 1, 2, 3, and 4 are represented by the identical histogram bars in all five panels of Fig. 5. The agreement for the human-to-human comparison between PHANGS-HST and LEGUS in NGC 3351 are 82, 53, 46, 73 per cent, with a mean value for the four classes of 64 per cent (shown as the 5th histogram bar).

These numbers for the human-to-human comparison in NGC 3351 are in somewhat better agreement (i.e. by 13 per cent in the mean) than the human-to-human comparison quoted by Wei et al. (2020), who compared agreement in source classifications between LEGUSBCW and the LEGUS 3-person-consensus for the galaxy NGC 4656. The slightly higher values we find here likely result from the same person classifying sources in NGC 3351, which reduces inherent bias which may exist between human classifiers. Our experience, both here and later in Table 1 , is that this is typically a $\sim 10$ per cent effect.

In Fig. 5, we also compare both the PHANGS-HST and LEGUS human source classifications in NGC 3351 with the machine learning classifications from RESNET and VGG. The agreement between the five different combinations which include at least one human classification (i.e. PHANGS-HST versus LEGUS, PHANGS-HST versus RESNET, PHANGS-HST versus VGG, LEGUS versus RESNET, and LEGUS versus VGG) are listed in Table 1 for NGC 3351. We find that in nearly all cases, the comparisons between the human
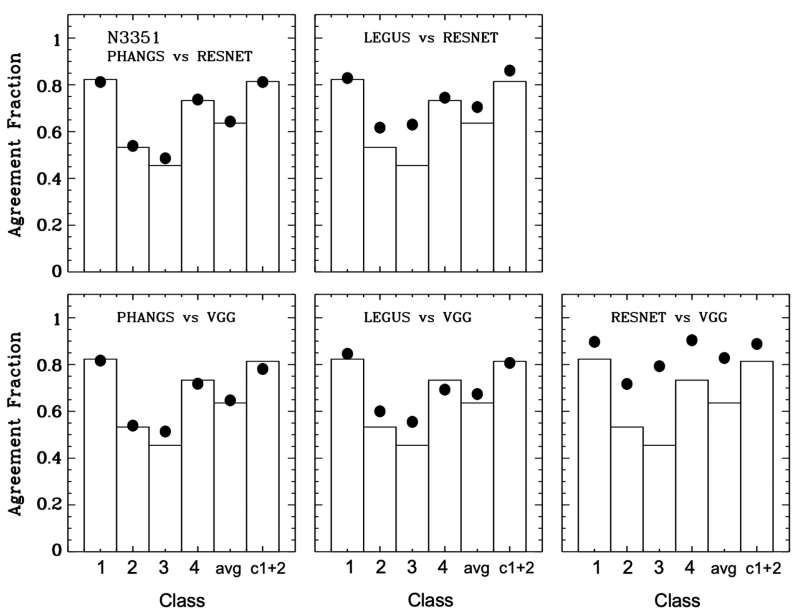

Figure 5. Comparison between classification for PHANGS-HST (human), LEGUS (human), RESNET, and VGG for NGC 3351, as described in text. The bars in the histogram, which are identical in all five panels, show the human-to-human baseline defined by comparing human classifications from PHANGS- $H S T$ and LEGUS. The filled circles show the agreement factions based on the comparison between the different studies, as denoted at the top of each panel. The fifth column shows the average of columns 1 to 4 , while the column labelled $\mathrm{c} 1+2$ shows the results when Classes 1 and 2 are considered as a single bin. Note that the filled circles, which include comparisons involving machine learning classifications, are generally the same as or above the histograms representing the PHANGS- $H S T$ versus LEGUS human-to-human comparison.

and machine learning classifications (i.e. the solid circles in Fig. 5) are as good or better than the human-to-human classifications (i.e. the histogram). We find the same general trends as in the humanto-human classifications, with the best agreement for Class 1 and the worst for Class 3. The mean for all five comparisons involving human classifications (i.e. leaving out the RESNET versus VGG classifications which are always higher) is 66 per cent, i.e. essentially the same as the mean for the human-to-human comparison in NGC 3351 being used as the benchmark (i.e. 64 per cent).

An additional bar (labelled ' $\mathrm{C} 1+2$ ') is included in Fig. 5 for the case where the Class 1 and Class 2 objects are combined into a single bin, a common procedure in many studies. We find an agreement fraction of 81 per cent for the baseline human-to-human (i.e. PHANGS-HST versus LEGUS) classifications for Class $1+2$ in NGC 3351, and similar values for all four of the other combinations in Fig. 5. These numbers are very similar to the agreement fractions for Class 1 alone.

The $\mathrm{C} 1+2$ sample will be considered the 'standard' sample in many aspects of the discussion throughout this paper. However, our general advice is to use both Class $1+2$ and Class 1 alone to see how they affect your science results, as we have done in Section 7.

Finally, we note the very high agreement fractions when the two machine learning algorithms (RESNET versus VGG) are compared (e.g. in the bottom right-hand panel in Fig. 5 for NGC 3351). We interpret this to be due to the repeatability when computer classification algorithms are used. However, there is no guarantee that the machine learning classifications are actually 'better' or 'correct', only that they are more repeatable. We shall revisit this point in Section 5 where we examine 'figures of merit'.

Table 1 also includes the agreement fractions in the four classes for all five galaxies studied here. We find that for all galaxies except NGC 628, the mean agreement fractions including the machine learning algorithms are higher than the PHANGS-HST versus 

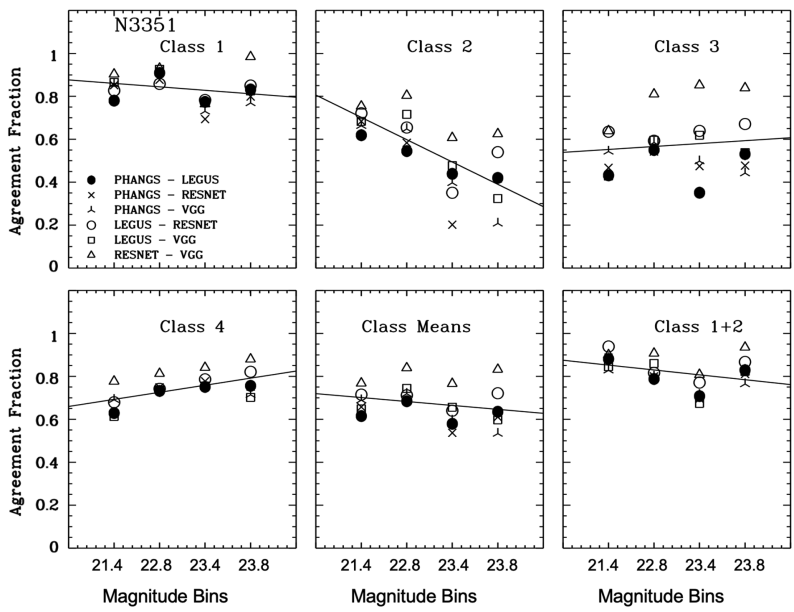

Figure 6. Agreement fractions as a function of magnitude bins for Classes 1 to 4 , the means of Classes 1 to 4 added together (bottom middle), and the values if Class $1+2$ are considered as one bin (bottom right-hand panel). The two studies being compared in each case are plotted with different symbols. Least-squares fits to all the data are shown by the lines. We find that while many of the dependencies are relatively flat (i.e. Class 1 , Class $1+2$ ) the agreement fractions for Class 2 falls rapidly as a function of magnitude.

LEGUS (human-to-human) ones. This, and the similar result for NGC 3351 shown in Fig. 5, are the primary reasons for our statement that the machine learning classifications are as good or slightly better than human classifications.

The two highest mean agreement fractions in Table 1 are for NGC 3627 (74 per cent) and NGC 3351 (66 per cent), which are the two galaxies classified by BCW in both PHANGS-HST and LEGUS. The slightly higher agreement, $\sim 10$ per cent, is likely for the same reason described earlier, that this reduces the systematic human-tohuman differences in classification methodology in this cases since the same human is involved, and the BCW LEGUS training set was used for RESNET and VGG classifications.

In general, there is no one study that appears to be much better than the others. For example, when considering the mean values from column 5 of Table 1 (excluding the RESNET versus VGG comparison), three different classifier combinations have the highest values for a given galaxy (i.e. PHANGS-HST versus VGG for NGC 628 and NGC 1566; PHANGS-HST versus RESNET for NGC 1433; and LEGUS versus RESNET for NGC 3351).

To summarize, we find that comparisons between all four classification methods give fairly similar results; all of them appear to provide source classifications of comparable quality.

\subsection{Agreement as a function of source brightness}

In Fig. 6, we examine how the results from different classifiers change with brightness, comparing the agreement fraction of sources in NGC 3351 in different magnitude bins. Classes 1, 2, 3, and 4 are shown in different panels. The symbols represent the different combinations of classifiers (PHANGS-HST, LEGUS, RESNET, VGG). The magnitude bins have been selected to contain roughly equal numbers of sources (of all classes), which leads to non-uniform size bins because there are many more faint clusters than bright ones. The medians of the bins are $m_{V}=21.4,22.8,23.4$, and 23.8 mag.

Probably the most important conclusion in this subsection is that the agreement fractions for Class 1 , and also for Class $1+2$ when considered one bin (i.e. the standard sample), are similar regardless of
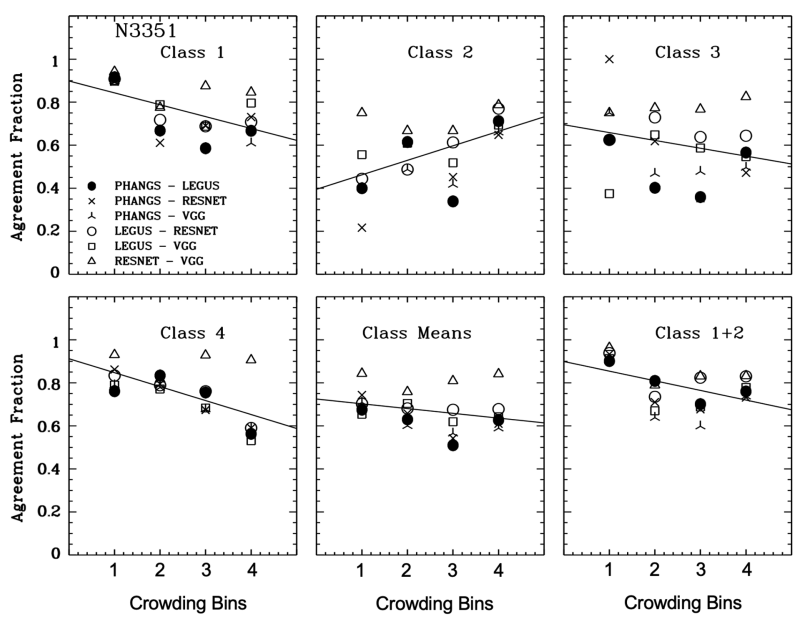

Figure 7. Agreement fractions as a function of crowding bins (bin $1=$ isolated, bin $4=$ most crowded) for Classes 1 to 4 , the means of Classes 1 to 4 , and Class $1+2$ considered as one bin. The two studies being compared in each case are plotted with different symbols. We find that most of the dependencies are steeper than versus magnitudes.

source brightness, with values around 85 per cent over the full magnitude range. This is reassuring since finding Class 1 and Class 2 clusters is the focus of our cluster classification effort. It also implies that we may be able to use the machine learning algorithms to push down to fainter magnitudes for these types of clusters. This is discussed in more detail in Section 5.6 and in D. Thilker et al. (in preparation).

Class 2 clusters alone, on the other hand, show a strong decreasing agreement fraction towards fainter magnitudes, starting around 70 per cent for bright clusters and dropping to about 40 per cent (with large scatter) at the faint end. This may reflect the fact that most bright Class 2 clusters are similar to Class 1, with some relatively minor asymmetries, while many of the fainter Class 2 clusters are more difficult to discriminate from Class 3 objects.

Note that the agreement fractions are generally higher when combining the Class 1 and 2 clusters into a single bin compared to averaging them together. This is because a frequent difference in classification is to interchange the two classes; i.e. to draw the line between symmetric and asymmetric slightly differently. Hence, some of the shortcomings discussed in this section and elsewhere in the paper for the classification of the Class 2 clusters alone are not as serious when combining the two into one bin.

The agreement fractions for Class 3 (compact associations) are essentially flat as a function of magnitude, albeit with a large scatter, while the agreement fractions for Class 4 (artefacts) actually increase at fainter magnitudes. The latter effect likely results because the fraction of contaminants in the form of individual and pairs of stars, which are relatively easy to classify, increases at fainter magnitudes.

\subsection{Agreement as a function of crowding}

In Fig. 7, we make comparisons for the agreement fractions as a function of source crowding. The methodology is essentially the same as for our comparisons with magnitudes shown in Fig. 6. The crowding parameter employed is from the DOLPHOT $V$-band 'crowd' parameter.

The catalogue is broken into four subsamples, with crowding bin $=$ 1 for isolated objects (e.g. old isolated clusters in the smooth bulge regions outside of the central starburst ring), to crowding bin $=4$ for 

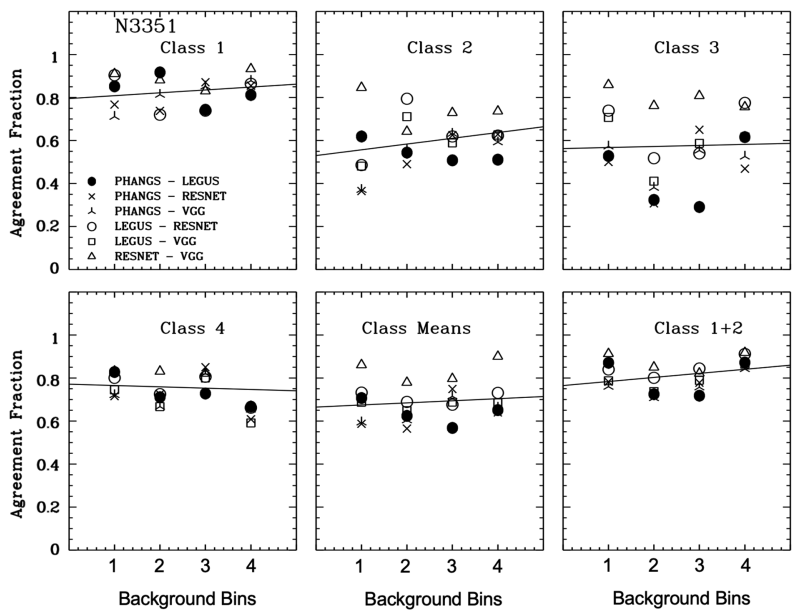

Figure 8. Agreement fractions as a function of local background bins (bin $1=$ low background, bin 4 = highest background) for Classes 1 to 4 , the means of Classes 1 to 4 , and Classes 1 and 2 together. The two studies being compared in each case are plotted with different symbols. We find that most of the dependencies are relatively flat.

very crowded regions (e.g. large associations and the central starburst ring).

The trends with crowding are as strong or stronger than those found with brightness. The results for each object class is shown in its own panel, as labelled in Fig. 7. We note the excellent classification agreement (mean $=92$ per cent) for isolated Class 1 objects (crowding bin $=1$ ), shown as the upper left points in the upper left-hand panel. These are primarily old clusters in the bulge which have had ample time to separate from their birth clouds and hence are generally isolated. The agreement falls to about 70 per cent for Class 1 for the more crowded bins. The reverse trend is seen for Class 2 objects, with the highest agreement fraction for the most crowded regions. This reflects the fact that crowded regions will often introduce apparent asymmetries in the outskirts of clusters, causing them to be classified as Class 2 (asymmetric clusters) rather than Class 1 (symmetric clusters). We note, however, that the relation for the standard Class $1+2$ sample, and the means, are relatively flat, though not as flat as versus magnitude. This indicates that crowding has a stronger impact than brightness on the classification of Class 1 and Class 2 objects, the main focus of this paper. Pérez et al. (2021) report similar issues with Class 2 and Class 3 classifications, hence this effect appears to be inherent in the difficulty of classifying these objects in crowded regions rather than in the particular machine learning classification method employed.

The scatter between the four different classification methods for some of the source types (e.g. Class 2 and Class 3) is large, presumably reflecting differences in how the different methods perform the classifications.

\subsection{Agreement as a function of local background}

In Fig. 8, we assess how much the background surface brightness affects the classification results, where the background is defined to be the median flux in the background annulus used for photometry (i.e. an annulus between 7 and 8 pixels in radius). In general, the agreement fractions do not vary significantly with background level, unlike the situation with magnitude and crowding. Part of the reason for this might be that some sources in regions of high background are found in the central region around the chaotic star formation

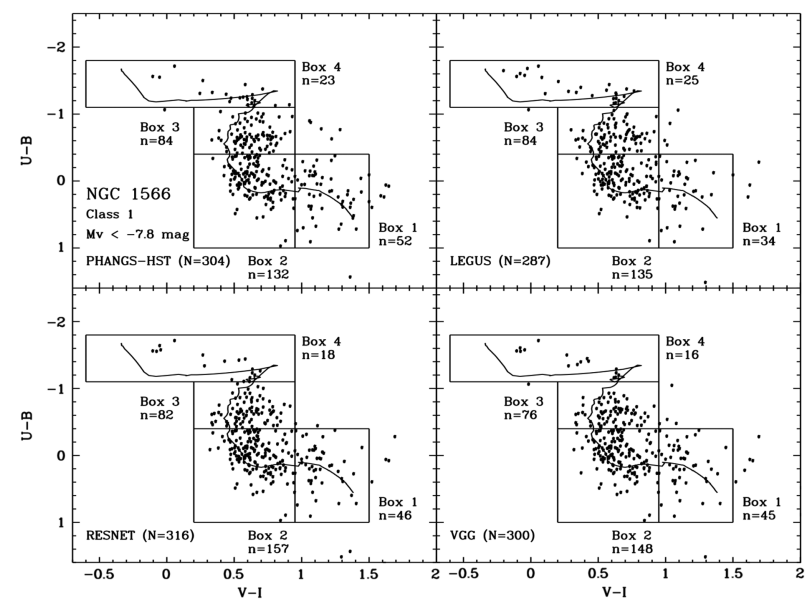

Figure 9. $U-B$ versus $V-I$ colour-colour diagrams for Class 1 objects for each of the four classification methods for $M_{V}<-7.8 \mathrm{mag}$. Four boxes corresponding to different age clusters are included, along with the number of objects in each box. A solar metallicity isochrone from Bruzual \& Charlot (2003) is included, running from $1 \mathrm{Myr}$ in the upper left to $10 \mathrm{Gyr}$ in the lower right. Note how similar the distributions of points are for all four of the classification methods.

ring. The agreement fractions are low here. Other sources with high background are in the smooth bulge component just outside of this region. The agreement fractions are actually high here. Hence, the results for these regions tend to balance each other out, resulting in relatively flat correlations.

\subsection{Agreement as a function of spatial resolution}

The agreement fractions are likely to be poorer for more distant galaxies, as it becomes more difficult to distinguish clusters from individual stars. However, it is difficult to test this hypothesis when we have only five galaxies in our sample. While there does appear to be a weak correlation in the expected sense (see Table 1), a larger sample will be required to make this determination in the future.

To summarize subsections 4.1 to 4.5 , crowding appears to introduce the largest uncertainties in the classifications, more so than faint magnitudes or high background. Hence, classification might be expected to be more problematic for galaxies with high star formation rates, producing large numbers of very young stars, which tend to both cluster more strongly resulting in higher crowding, and produce higher backgrounds.

\section{CLASSIFICATION ACCURACY USING COLOUR-COLOUR DIAGRAMS}

While we make relative rather than absolute comparisons throughout most of this paper, we also develop 'figures of merit' to help guide us toward the best methods of classification. One straightforward approach is to use locations of measured clusters in colour-colour plots.

Fig. 9 shows the U-B versus V-I diagram in NGC 1566 based on the four different classification methods. Only Class 1 objects are shown. This colour-colour plot will be used throughout Section 5. Fig. 10 shows another version of the colour-colour plot, but substitutes the $U V$ filter for the $U$ band. We focus on NGC 1566 as an illustrative example; results for all five galaxies are included in Table 2. The results for all the galaxies are similar, as will be shown in Section 5.5. 


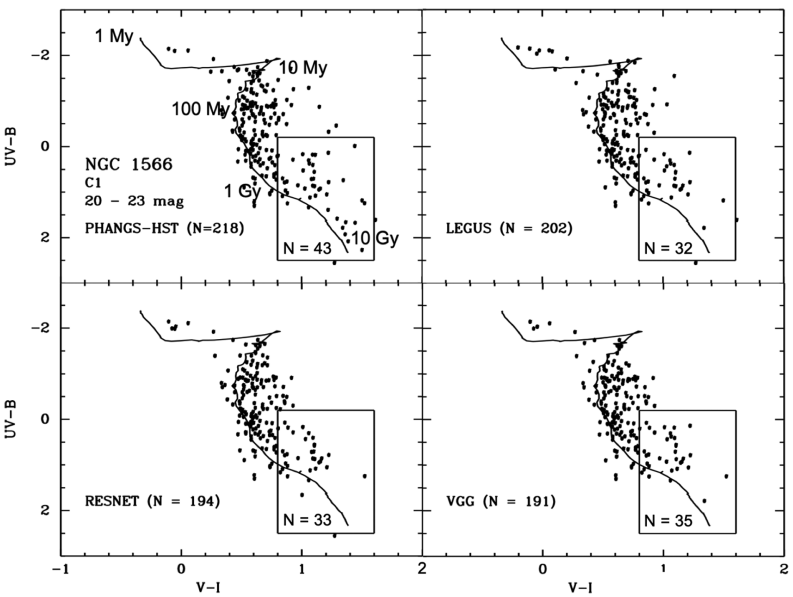

Figure 10. $U V-B$ versus $V-I$ colour-colour diagrams for Class 1 objects for each of the four classification methods for $m_{V}=20-23$ mag. Ages for positions along the isochrone have been added in the upper left-hand panel. A box appropriate for old globular clusters is included.
In these colour-colour diagrams, measured cluster colours (data points) are compared with predictions from the solar metallicity (Bruzual \& Charlot 2003) cluster evolution models (lines). The colours of clusters change with age, as the most massive (generally blue) stars die off. This predicted evolution through colour-colour space is highlighted in the upper left-hand panel of Fig. 10, where the predicted ages are shown, starting at $1 \mathrm{Myr}$ in the upper left-part of the diagram and ending at $10 \mathrm{Gyr}$ in the lower right.

Several papers demonstrate how the colour distributions of the sources change with morphological type (e.g. Adamo et al. 2017; Whitmore et al. 2020; Turner et al. 2021), since older clusters tend to be both redder and more symmetric. This is clearly observed in Fig. 9 by the lack of clusters along the youngest portion of the cluster evolutionary track. Not surprisingly, the same lack of very young clusters is found in Fig. 10. Class 2 clusters are asymmetric by definition, typically because they are in more crowded regions or because the cluster has not had sufficient time to dynamically relax, and we therefore expect them to be younger than Class 1 objects, i.e. to have colours indicative of younger ages. Fig. 11 shows the distributions for Class 2 objects. A significant fraction of these objects have colours suggesting they are 10-100 Myr old. There are also a relatively large number of Class 2 objects with colours

Table 2. Colour-colour statistics for four classification methods.

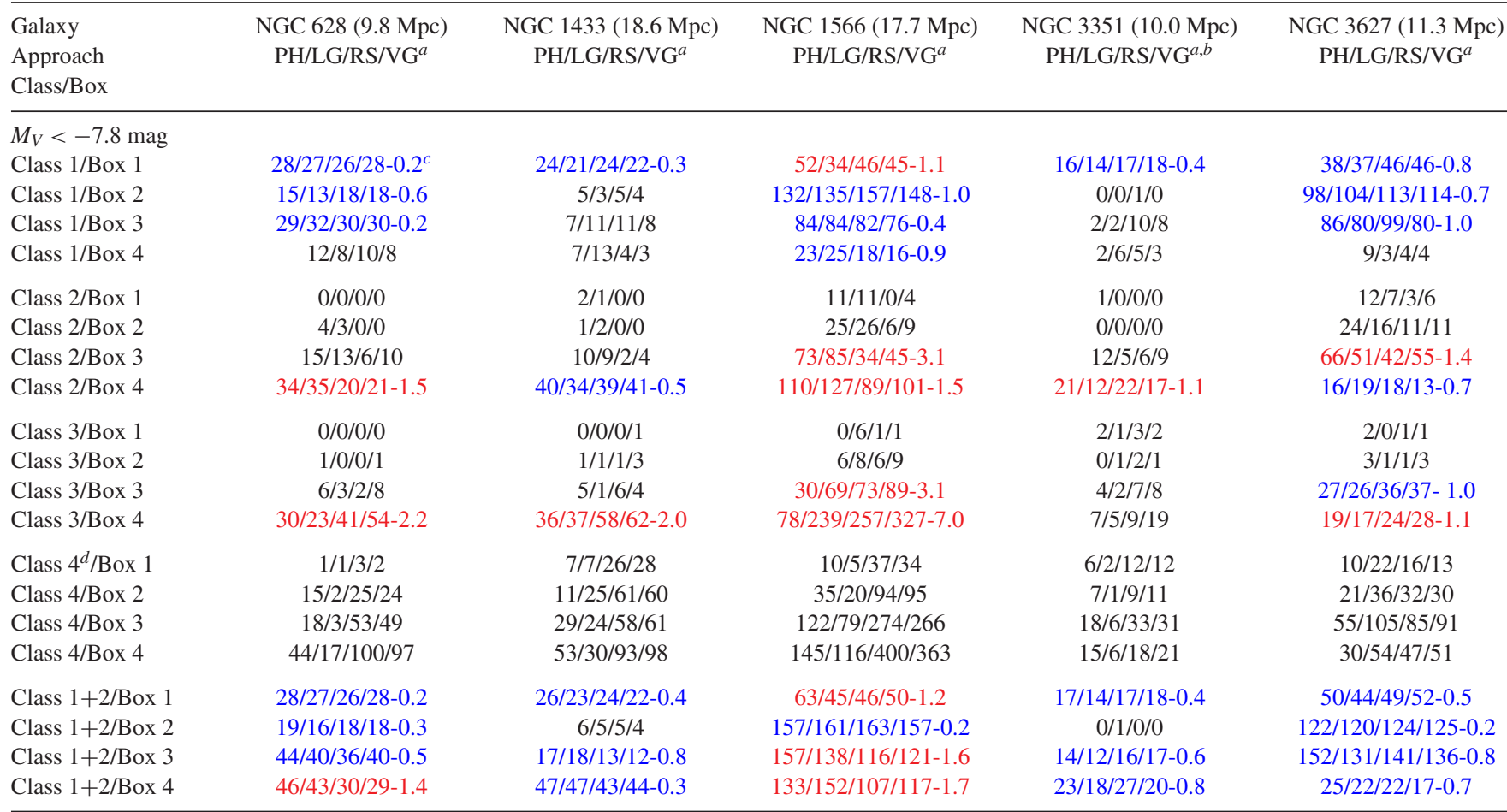

Notes. The boxes are defined as:

Box 1 - Old: $0.95<V-I<1.5$ and $-0.4<U-B<1.0$

Box 2 - Intermediate: $0.2<V-I<0.95$ and $-0.4<U-B<1.0$

Box 3 - Young: $0.1<\mathrm{V}-\mathrm{I}<0.95$ and $-1.1<U-B<-0.4$

Box 4 - Very young: $-0.6<\mathrm{V}-\mathrm{I}<0.95$ and $-1.8<U-B<-1.1$

${ }^{a} \mathrm{PH}=$ PHANGS $-H S T, \mathrm{LG}=$ LEGUS, RS $=$ RESNET, VG $=$ VGG.

${ }^{b}$ The NGC 3351 LEGUS field of view was roughly 30 per cent smaller than the fields of view for the other studies. Only the overlapping fields of view were used to calculate the colour-colour statistics.

${ }^{c}$ The values after the hyphens are the Quality Ratios $(\mathrm{QR})$, defined as the standard deviation of the four measurements divided by the square root of the mean number of objects in the box. Only values with all four columns having more than 12 measurements are included.

Numbers in blue have 'good' agreement (i.e. $\mathrm{QR}<1$ ). Numbers in red have 'poor' agreement (i.e. QR $>1$ ).

${ }^{d}$ Class 4 was not used in the analysis since the machine learning methods used a larger empirical selection region of the MCI plane, resulting in much larger numbers of artefacts. This would skew the value of QR if included. 


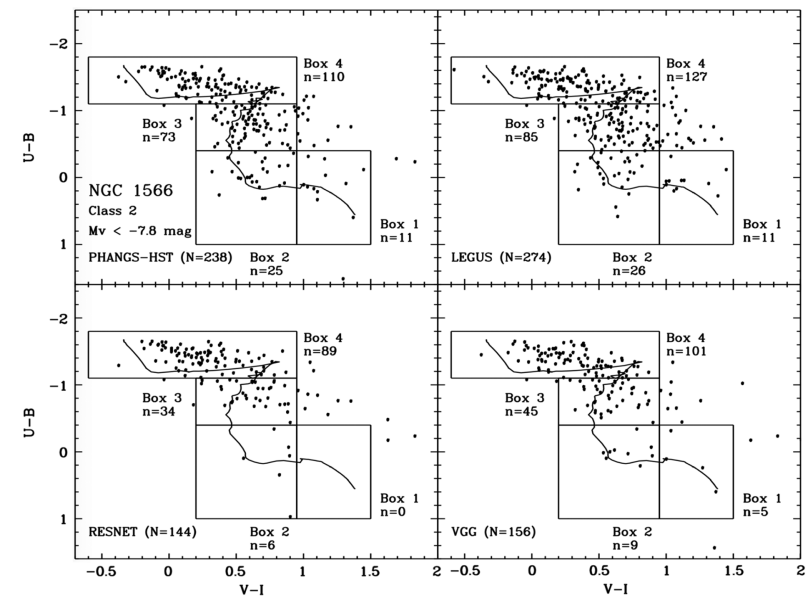

Figure 11. Same as Fig. 9, but for Class 2 objects. Note the lower numbers of objects in Box 3 for RESNET (34) and VGG (45) relative to PHANGS-HST (73) and LEGUS (85).

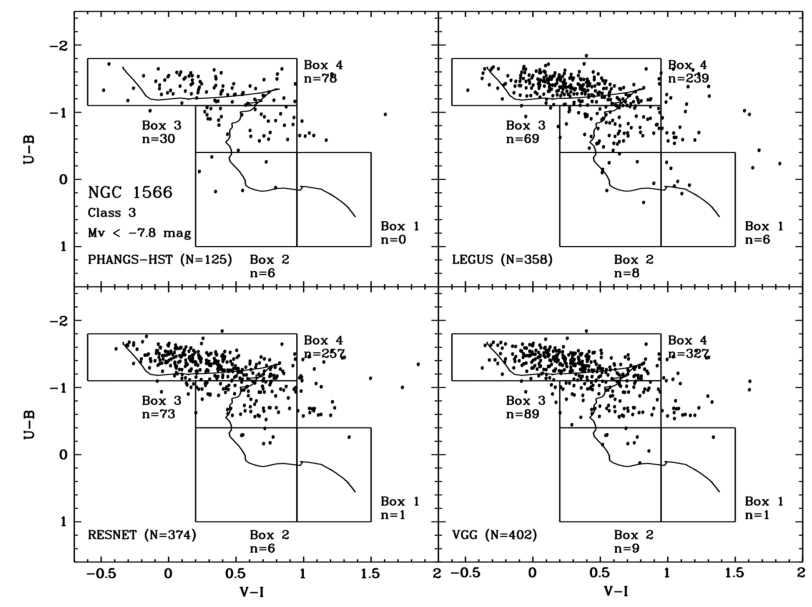

Figure 12. Same as Fig. 9, but for Class 3 objects. The lower numbers of PHANGS-HST Class 3 clusters is expected since the MCI method of selecting candidate clusters is designed to reduce the number of Class 3 (compact associations) and Class 4 (artefacts) (see D. Thilker et al., in preparation).

indicating they are younger than $10 \mathrm{Myr}$ (i.e. with $U-B$ colours bluer than $U-B=-1.0)$. Therefore, this correlation provides a way to test between Class 1 versus Class 2 clusters, i.e. most old, red clusters should be identified as Class 1, while few old, red clusters should be identified as Class 2.

Fig. 12 shows the distributions for Class 3 objects, which are generally found to be bluer and hence have younger ages (i.e. $<10 \mathrm{Myr}$ ), with typical values of $V-I$ between -0.6 and 1.0 , and $U-B$ less than -1.0 . There are also a sprinkling of Class 3 objects that have redder colours, likely due to a combination of age, reddening, and stochasticity effects (see Maíz Apellániz 2009; Fouesneau et al. 2012; Hannon et al. 2019; and Whitmore et al. 2020). As discussed in these papers, stochasticity is primarily an issue for lower mass clusters, i.e. with $\log$ mass $<3.5$ solar masses.

Hence, it appears that the distribution of data points in the colourcolour diagram can be used as a 'figure of merit' for our cluster classifications. Below we make quantitative checks to test the quality and uniformity of the different classification methods.

Figs 9, 11, 12, and 13 also include four boxes drawn to roughly demarcate different ages in the U-B versus V-I colour-colour

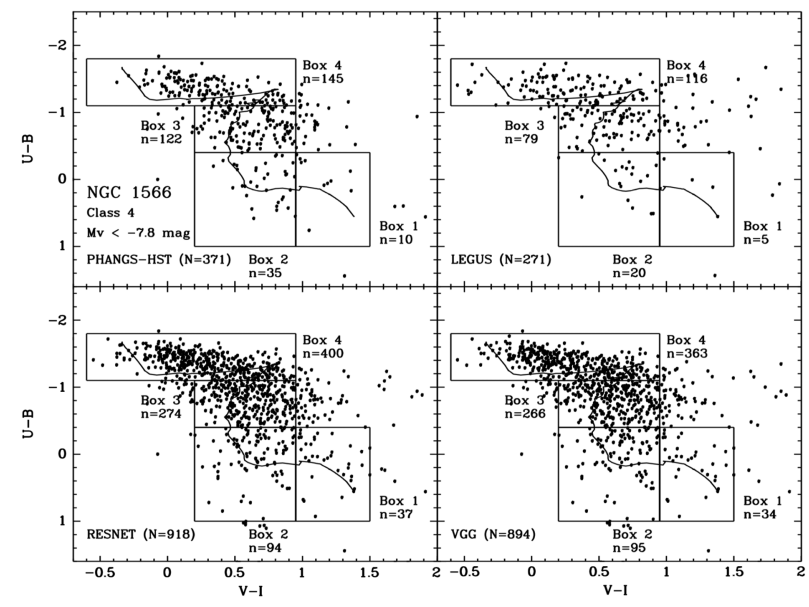

Figure 13. Same as Fig. 9, but for Class 4 objects. The larger numbers of RESNET and VGG Class 4 objects (artefacts) is primarily due to the use of the larger model selection region in the MCI plane for the two machine learning methods, rather than the smaller empirical polygon selection region used to identify candidates for the human PHANGS-HST classification (see D. Thilker et al., in preparation, for details). This demonstrates that a much higher percentage of the objects in regions outside the polygon are artefacts, as expected.

diagram, as defined below. This procedure is similar to an approach originally used in Whitmore et al. (2010) to divide the colour-colour plane into regions for the purpose of separating stars and clusters in the Antennae galaxies (also see Chandar et al. 2010b for a similar treatment in M83).

The regions are defined as:

Box 1: Old clusters ( $>1$ Gyr): $0.95<V-I<1.5$ and $-0.4<U-B$ $<1.0$

Box 2: Intermediate-age clusters (0.1 to $1.0 \mathrm{Gyr}): 0.2<V-I<$ 0.95 and $-0.4<U-B<1.0$

Box 3: Young clusters (10-100 Myr): $0.2<V-I<0.95$ and -1.1 $<U-B<-0.4$

Box 4: Very young clusters $(<10 \mathrm{Myr}):-0.6<V-I<0.95$ and $-1.8<U-B<-1.1$

We note that when mapping the regions of the colour-colour diagram on to apparent ages we assume no reddening. This is a reasonable assumption for objects older than $10 \mathrm{Myr}$ (see Whitmore et al. 2020), but it should be kept in mind that these age estimates are approximate. Values for the number of clusters in each box for each classification method are included in Table 2.

Examining Fig. 9, we first note that the total number of Class 1 objects (symmetric clusters) classified by the four methods are similar, with 304, 287, 316, and 300 objects from box 1 to box 4. We note the strong similarity in the overall colour distributions for Class 1 objects for all four methods, each hugging the right-hand side of the stellar isochrone in the region from $10 \mathrm{Myr}$ to about $1 \mathrm{Gyr}$, and then broadly following the isochrone itself in the region beyond 1 Gyr. Fig. 10 shows the same using the UV instead of the $U$ filtre, although the old globular clusters tend to be somewhat high. This is because we show a solar metallicity isochrone which is appropriate for the young but not the oldest clusters (see Turner et al. 2021, for a discussion). The strong similarity between the four panels in Figs 9 and 10 indicates that all four of the methods are finding similar Class 1 objects, which is very reassuring.

Fig. 11 includes the relative distributions within the four boxes for Class 2 objects (asymmetric clusters). The distributions of cluster colours in these four panels look less similar than for Class 1, with a 
Table 3. Breakdown of classes and subclasses for the five program galaxies using PHANGS-HST classifications.

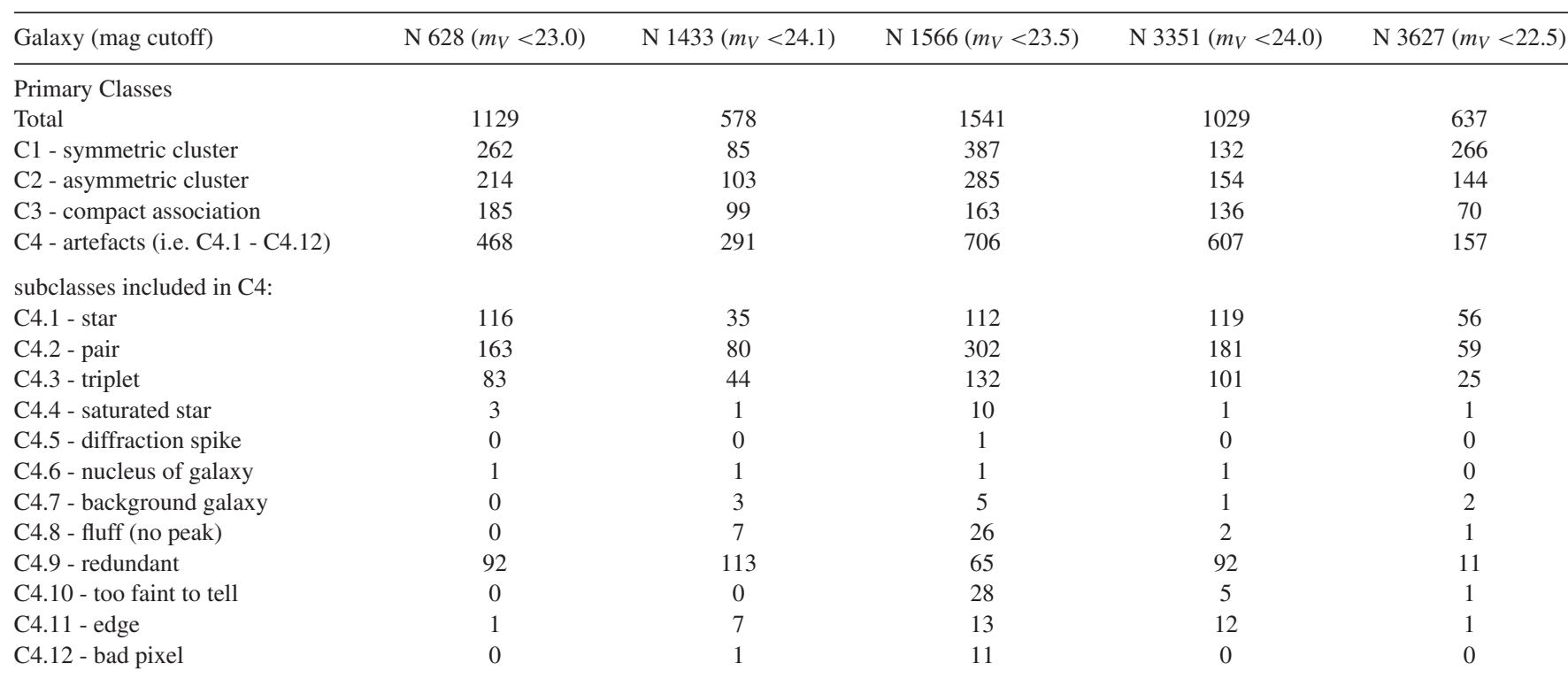

Notes. 1. For the NGC 3627 mosaic, only the southern field which overlaps with LEGUS is used. The full human PHANGS-HST catalogue has 1368 objects in it.

2. For NGC 1566, the whole field has been humanly classified to at least $m_{V}=23.5 \mathrm{mag}$, but parts of the image have been spot checked to fainter magnitudes.

clear deficit in Box 3 based on RESNET and VGG classifications (34 and 45) relative to the PHANGS-HST and LEGUS classifications (73 and 85). This suggests that the machine learning algorithms do not identify as many young objects (Box 3) as Class 2 when compared with PHANGS-HST and LEGUS.

Fig. 12 shows the results for Class 3 sources (compact associations). The most obvious difference is the much smaller number of classified objects in PHANGS-HST. This is largely by design, since the MCI method discussed in Section 3.2 was introduced to minimize the number of Class 3 (compact associations) and Class 4 (artefacts) objects. In addition, Class 4 has been broken into several subclasses (see Table 3), in an attempt to allow the machine learning algorithms to better classify (and remove as artefacts) similar objects in future treatments. Hence, pairs and triplets are included as Class 4 objects (artefacts). Since LEGUS used a somewhat looser definition for its Class 3 , it includes a fair fraction of pairs and triplets, most of which would be included as part of the expanded Class 4 in PHANGSHST rather than as Class 3 objects. If we add the Class 4.2 (pairs) and Class 4.3 (triplets) objects to Class 3 for PHANGS-HST, the number would increase from 125 to 345 , roughly the same as found for LEGUS (i.e. 358).

Fig. 13 shows the results for Class 4 sources. The most obvious difference is the larger number of classified objects for RESNET and VGG compared with human classification. This is primarily due to the selection of candidates from the larger region in the MCI plane for the two machine learning methods, rather than the smaller empirical polygon selection region used to identify candidates for the human PHANGS-HST classifications. See D. Thilker et al. (in preparation) for a description of the candidate selection procedure for PHANGSHST. The larger number of Class 4 objects for RESNET and VGG demonstrates that a much higher percentage of objects in regions outside the polygon in the MCI plane are artefacts, as expected.

The distribution of Class 4 points in the colour-colour plots is most similar to the Class 2 objects, but with a wider spread due to the stochasticity imposed by the low number of stars in the objects (i.e. $\mathrm{c} 4.2=$ pairs and $\mathrm{c} 4.3=$ triplets are the most populated subclasses).

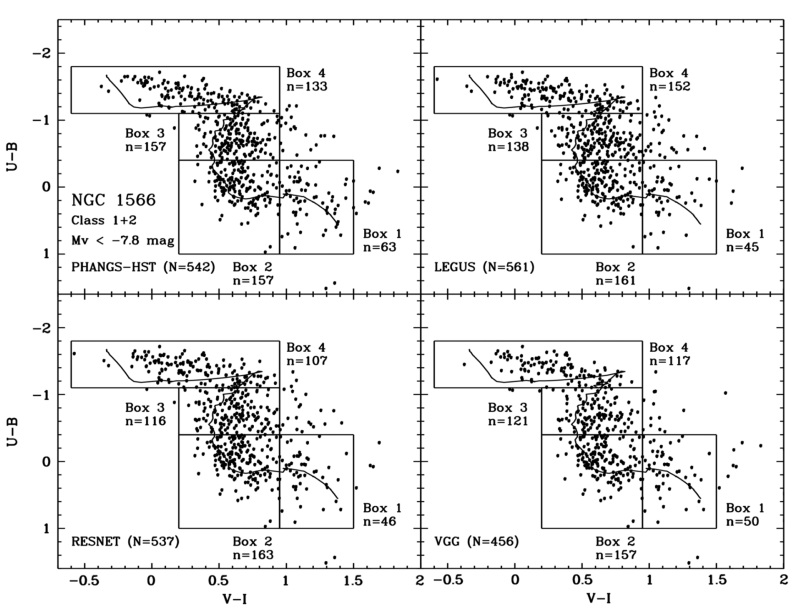

Figure 14. Same as Fig. 9, but for Class $1+2$ objects (i.e. the 'standard' sample.) Note how similar the distributions of points are for all four of the classification methods, similar to the results for Class 1 in Fig. 9, but with a larger number of clusters.

We also note a small increase in the percentage of objects in Box 1 (old clusters) for RESNET and VGG (3.7 per cent and 3.6 per cent, respectively) compared to PHANGS and LEGUS (2.5 per cent and 2.1 per cent, respectively). This suggests that the machine learning algorithms misclassify a slightly larger fraction of old globular clusters as artefacts.

Fig. 14 shows the results for Class $1+2$ sources included together. As discussed in Section 4.1, combining these two classes (where the primary criteria is central concentration) is a common practice, although we also suggest experimenting with Class 1 alone to see how the science results of a given project might be affected. In Section 6.3.2, we will find that Class 1 and Class 2 are frequently interchanged, due to slightly different estimates of the degree of asymmetry. Hence combining the two classes results in agreement 

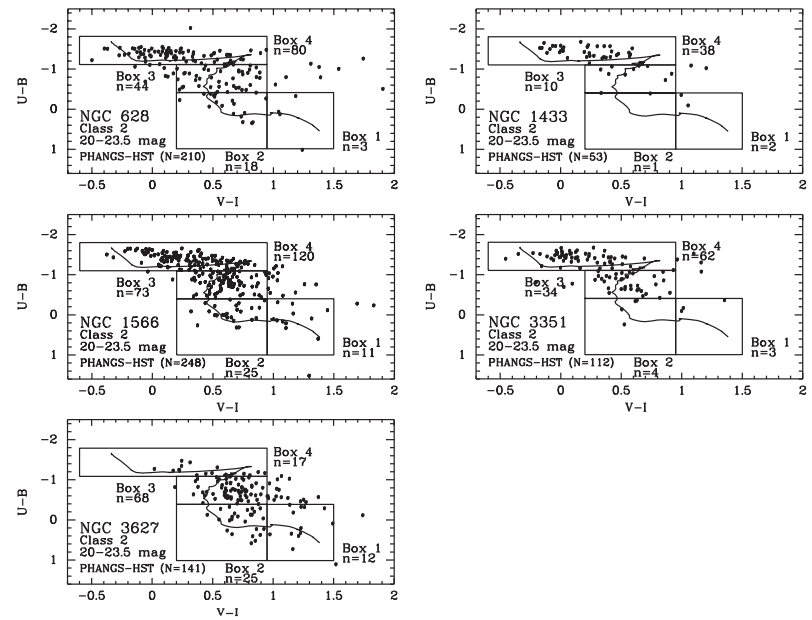

Figure 15. Same as Fig. 9 for Class 2 clusters for all five program galaxies in range $m_{v}=20-23.5 \mathrm{mag}$. We find a wide variety of different cluster formation histories from NGC 1433, with 72 per cent of the objects in the youngest box, to NGC 3627, with 12 per cent of the objects in the youngest box.

fractions and other properties that are roughly as good or slightly better than Class 1 alone in most cases. For these reasons, we call Class $1+2$ the 'standard' cluster sample in this paper.

We first note that like Class 1 , the distributions are very similar for all four classification methods in NGC 1566 . Hence all the methods find similar objects. While Class 1 alone has very few young objects, Class $1+2$ has a more even distribution of ages, which can be a useful characteristic for many science projects. VGG has marginally fewer Class $1+2$ clusters than the other three methods, with a slight tendency to find more Class 3 objects, as can be seen in Fig. 12. A similar but weaker tendency is seen for the other galaxies, as shown in Table 2.

One of the primary results based on examining Figs 9 to 13 is that all four methods of classification give fairly similar results for NGC 1566. This will be addressed in more detail in Section 5.5. However, one surprise is that the distribution of Class 2 objects looks more like the Class 3 objects than the Class 1 objects in NGC 1566, unlike previous results such as for NGC 4449 (Whitmore et al. 2020). One possible explanation is that the galaxies have different cluster formation histories.

In Fig. 15, we show the Class 2 colour-colour diagrams for all five galaxies. We find a fairly wide range in cluster distributions from NGC 1433 with 72 per cent of the points in the youngest box to NGC 3627 with only 12 per cent in the youngest box. NGC 1566 is intermediate with 48 per cent. Hence, NGC 3627 looks more similar to NGC 4449, and we conclude that the degree of similarity between Class 2 and 3 is largely dependent on the cluster formation history. Table 2 provides similar statistics for all four classes and all five program galaxies. We now perform four specific tests, as motivated above.

\subsection{Old clusters ( $>1$ Gyr)}

For this test, we ask how many Class 1 objects are found in Box 1 for each classification approach.

Fig. 9 shows that using the galaxy NGC 1566 as our test case, the $U-B$ versus $V-I$ diagram, and $M_{V}<-7.8$ mag, PHANGS $-H S T$ finds the most Class 1 objects in Box 1 (old clusters), with 52, compared to 34, 46, and 45 for LEGUS, RESNET, VGG, respectively. If the UV-B versus $V-I$ diagram is used instead, and a range of $m_{V}$ from 20 to 23 as

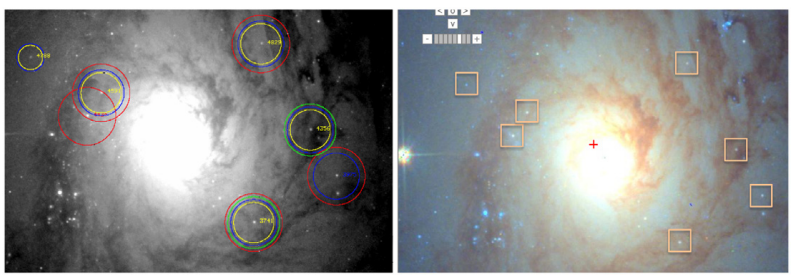

Figure 16. Left-hand panel shows the F555W image in the inner region of NGC 1566, with all the Class 1 clusters from Box 1 in this field of view identified. Red $=$ PHANGS- $H S T$, green $=$ LEGUS, blue $=$ RESNET, yellow $=$ VGG. The right-hand panel shows a colour image using F814W, F555W, and F438W, with squares around the locations of the clusters.

in Fig. 10, the numbers are 43, 32, 33, 35, respectively. We conclude that all four methods are able to find old clusters at similar levels.

Fig. 16 shows an image of the old cluster candidates (i.e. Class 1 objects in Box 1) in the nuclear region of NGC 1566. This bulge region has the highest density of Class 1 objects from Box 1, as would be expected if they are old globular clusters. Red is used for PHANGS-HST, green is used for LEGUS, blue is used for RESNET, and yellow for VGG. The larger circles show sources in the range $m_{V}=20$ to $23.5 \mathrm{mag}$ while the smaller circles represent sources in the range $m_{V}=23.5$ to $24.5 \mathrm{mag}$. One of the objects (3741) is found by all four of the classifications. Three more of the objects are found by three of the four methods.

A visual examination of the right-hand panel of Fig. 16 shows the objects all have fairly uniform yellowish colours and symmetric morphologies. Hence, these are all good candidate old globular clusters, demonstrating that all four methods are able to identify this type of object fairly successfully.

We also note from Fig. 11 that Class 2 includes very few objects in Box 1 for all four classification approaches (i.e. 11, 11, 0, 5 for PHANGS-HST, LEGUS, RESNET, VGG, respectively), and from Fig. 12 we find even fewer $(0,6,1,1)$ in Box 1 for Class 3 . Hence the rejection of the oldest clusters for Classes 2 and 3 is also good.

\subsection{Intermediate age clusters $(0.1-1$ Gyr $)$}

For this test, we ask how many Class 1 objects are found in Box 2 of colour-colour space for all four approaches.

From Fig. 9, we find that the RESNET and VGG classification methods identify somewhat more symmetric, intermediate-age clusters (i.e. 157 and 148 objects in Box 2) than PHANGS-HST and LEGUS (132, and 135 in Box 2). We note, however, that most of this shortfall for Class 1 objects in Box 2 shows up as higher numbers in Class 2 for PHANGS-HST and LEGUS (i.e. Fig. 11, with 25 and 26 for PHANGS-HST and LEGUS, respectively, compared with 6 and 9 for RESNET, and VGG, respectively).

\subsection{Young clusters (10-100 Myr)}

A similar approach can be used for the objects in Box 3, but here we add the results from Classes 1,2, and 3 since they are spread out more in the different classes. We find that LEGUS and VGG find the most young objects (238 and 210) in Box 3, while RESNET has 189. The largest numbers of objects in Box 3 are found in Class 1, with a small spread ranging from 76 to 84 for all four methods (see Fig. 9).

\subsection{Very young clusters (1-10 Myr)}

For our Box 4 comparison we only use Class 2 clusters since there are only a handful of very young clusters in Class 1 for all four 

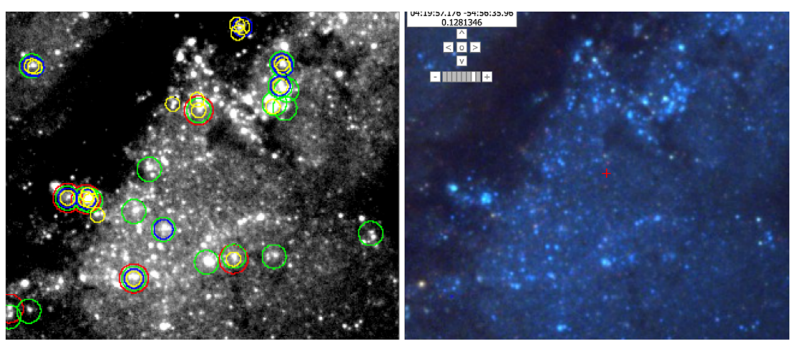

Figure 17. Same as Fig. 16, but for Class 3 compact associations. Note that nearly all of the Class 3 objects, selected based on their morphology, are bluish in colour as expected for very young objects. There are fewer PHANGS-HST (red) Class 3 objects, as discussed in the text.

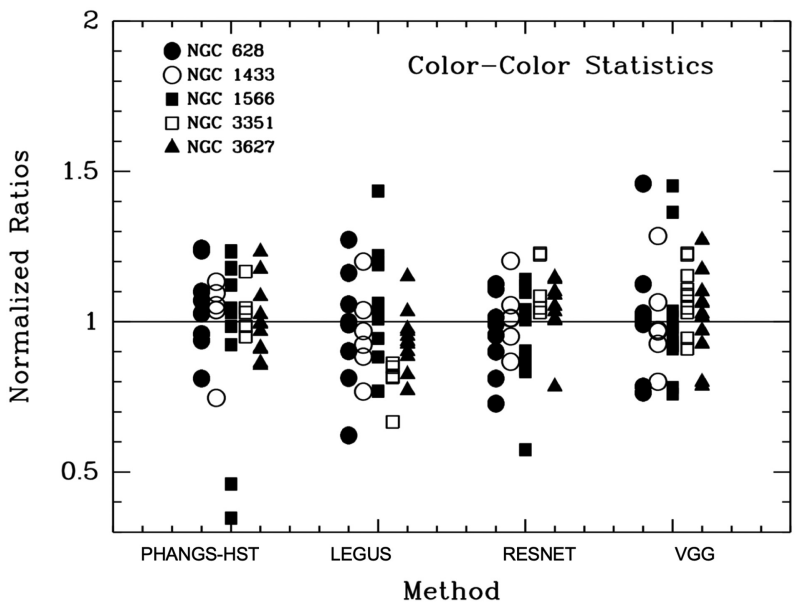

Figure 18. Figure of merit measurements using the number of objects in colour-colour diagram boxes from Table 2 . Only cases where all four methods have at least 12 objects within the box are included. Values are normalized by the mean for the four methods. Class 4 comparisons are not included.

methods and the rejection of most of the Class 3 objects using the MCI method makes inclusion of PHANGS-HST problematic. The agreement between the four methods of classification is fairly good, with values of 110 and 127 for PHANGS-HST and LEGUS and slightly lower values of 89 and 101 for RESNET and VGG. Hence, the machine learning algorithms and human classifications are finding fairly similar objects for the very young objects in Box 4 .

Although the focus in this paper is on the clusters, and to a lesser degree on compact associations (see K. Larson et al., in preparation, for the preferred approach to working with associations in PHANGS$H S T)$, we note from Fig. 13 that the most populated box for Class 4 (artefacts - i.e. single stars, pairs, triplets etc) is Box 4, indicating that individual stars as well as young clusters can be found in Box 4. Indeed, the left half of Box 4 was called 'star/cluster space' in Chandar, Fall \& Whitmore (2010a). Fig. 17 shows an image with comparisons of the Class 3 compact associations identified for the four classification methods. There are fewer PHANGS-HST Class 3 objects, as discussed later in the text.

We conclude that all four of the classification methods result in fairly similar distributions in the $U-B$ versus $V-I$ colour-colour diagrams. However, there are also some important second-order differences that should be kept in mind, for example, RESNET and VGG find fewer young (Box 3) clusters in Class 2 than PHANGSHST and LEGUS.
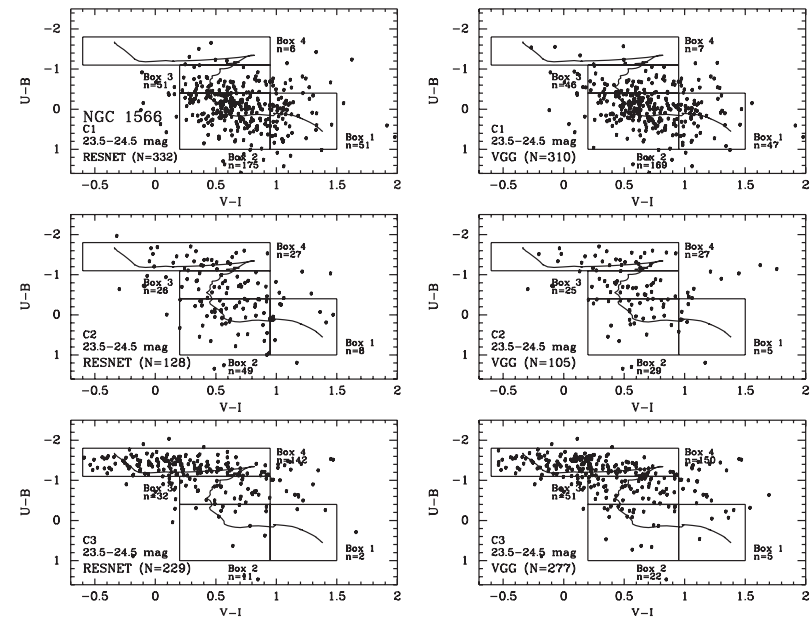

Figure 19. $U-B$ versus $V-I$ colour-colour diagrams for Classes 1 (top), 2 (middle), and 3 (bottom) objects for the RESNET (left) and VGG (right) classifications. Only faint clusters in the range $m_{V}=23.5$ to $24.5 \mathrm{mag}$ are included, i.e. fainter than for most of the PHANGS-HST and LEGUS human classifications. Four boxes representing different ages are included, along with the number of objects in each box. While these figures are similar to Figs 9, 11, and 12 for brighter clusters in some regards (e.g. the ability to find old clusters in Box 1), they also show important difference, such as fewer numbers of Class 2 and Class 3 clusters.

\subsection{Using colour-colour statistics as a figure of merit}

Similarities in the agreement fractions between the four classification methods, as discussed in Section 4.1, provides evidence that the machine and human classifications are similar in performance. In this section, we take a more quantitative look by comparing the colourcolour statistics for all four methods and all five galaxies. This 'figureof-merit' provides both absolute and relative comparisons of how well the different methods are able to identify different age clusters.

Table 2 includes the number of objects in each of the four boxes in the colour-colour diagram for all five galaxies. A limiting magnitude of $M_{v}=-7.8 \mathrm{mag}$ is used to normalize the sample. This is the magnitude cutoff of $m_{v}=22.5$ for the PHANGSHST human selected sample for NGC 3627, which represents the brightest limiting magnitude of the five galaxies. A value of $m_{v}=$ 22.5 mag provides a signal-to-noise of $\sim 100$, and represents a very conservative limit (see S. Deger et al., in preparation) for classification. A magnitude cutoff of $m_{v}=23.5$ corresponds to a range of $M_{V}=-6.5$ to -7.8 mag for our galaxies, and represents a more standard limit. Pushing to $m_{v}=24.5 \mathrm{mag}$, as we will in Section 5.6, corresponds to a signal-to-noise around 10, and should be considered the practical limit. When all four methods have 12 or more sources in Table 2 we check to see how constant the values are, i.e. do the different methods find similar numbers of clusters in the same regions of the colour-colour diagram?

To make the comparison, we use the ratio between the standard deviation of the four measurements and the square root of the mean number of objects in the box (i.e. the predicted standard deviation assuming Poisson statistics), which we define as the Quality Ratio = QR. These numbers are included at the end of the rows that qualify for analysis in Table 2. Measurements with values 1.0 or lower (i.e. fairly constant values) are shown in blue, while measurements with values greater than 1.0 (i.e, discrepant values) are shown in red in Table 2.

Class 4 objects (artefacts) are not included in the analysis since the goal of the PHANGS-HST and LEGUS studies was to eliminate as many artefacts as possible using constraints on either the 


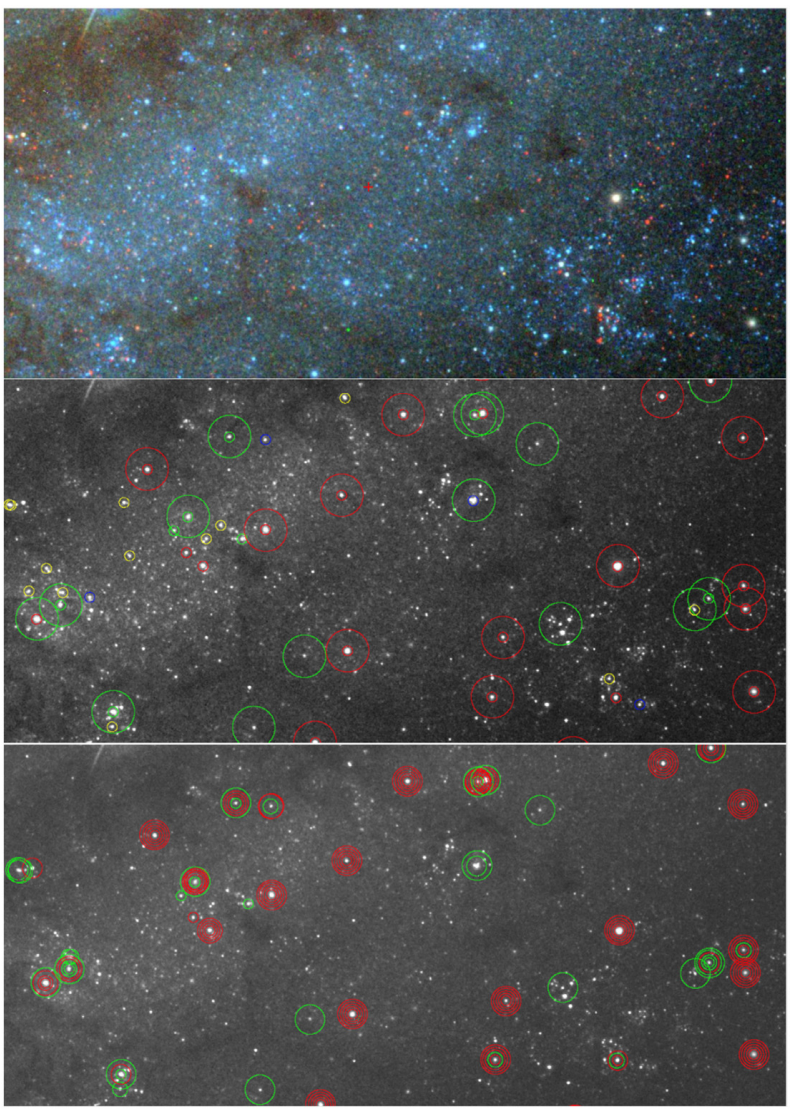

Figure 20. A search for 'missing' clusters and a comparison of the classifications for different methods for a field in NGC 628. The top panel shows a colour image from the HLA interactive display tool - https: //archive.stsci.edu/hlsp/phangs-hst. We suggest that readers electronically enlarge the image; pick out objects they think are clusters, and then compare with the panels below. The middle panel shows Class 1 (red) and Class 2 (green) clusters from the human-selected cluster classification (large circles) and the PHANGS-HST catalogues (small circles). Class 3 (blue - compact associations) and Class 4 (yellow - artefacts) are also included for PHANGSHST. The bottom panel uses the same colour scheme but includes all five classifications from PHANGS-HST (smallest circles), to LEGUS, to RESNET, to VGG, to the human-selected classifications (largest circles).

concentration index (LEGUS) or on multiple concentration indices (PHANGS-HST). In addition, the machine learning algorithms used much broader constraints since the time required to make the classification is not a factor, unlike for the human classifications. These two effects result in much larger numbers of Class 4 artefacts for RESNET and VGG, which skew the QR statistic. This increase in the number of Class 4 objects for the machine learning algorithms can be seen in Fig. 13. The result is that all Class 4 QR values are very high, by design, but this is not relevant for a comparison of the goodness of the different classification methods. Similar statements could be made for the Class 3 objects, as shown in Fig. 12, since the PHANGS-HST candidate selection is designed to eliminate as many of these objects as possible. However, since the discrepancies are smaller in most cases, and there is more interest in Class 3 (compact associations) than Class 4 (artefacts) objects, they have been included in the analysis for the purpose of comparison. We find, as expected, that most of the Class 3 objects have QR values greater than 1.0 in Table 2.

The results of the figure of merit analysis are shown in Fig. 18. We find that all four methods are successful in finding similar numbers of objects based on the colour-colour statistics, with mean values and standard deviation values for PHANGS-HST $=1.01 \pm 0.18$, LEGUS $=0.97 \pm 0.16$, RESNET $=1.00 \pm 0.14$, and VGG $=$ $1.02 \pm 0.17$. Similarly, values for the different galaxies are roughly the same, with no clear outliers in the mean.

Twenty-eight rows in Table 2 have values of $\mathrm{QR}=1.0$ or lower (i.e. good agreement, blue) while fifteen rows have values greater than $\mathrm{QR}=1$ (i.e. poor agreement, red). This shows that while the four methods find similar objects in the majority of cases, there are also a number of cases where systematic differences are present. A close look at Table 2 shows that the primary causes for large values of QR are either smaller number of Class 3 associations, as designed into the PHANGS-HST selection criteria, or the smaller number of young (Box 3 and Box 4) Class 2 objects found by RESNET and VGG, as shown in Fig. 11 and discussed in Section 5.

\subsection{Results for faint objects using machine learning classifications}

An important advantage of using machine learning classifications is the opportunity to provide cluster catalogues that include fainter objects, since classifying large numbers of sources is no longer prohibitive. Below we investigate how accurately fainter clusters can be classified by comparing the numbers of sources found in the different regions of colour-colour space with their brighter counterparts.

Fig. 19 shows the colour-colour plots for RESNET and VGG classifications of Class 1,2, and 3, with $m_{V}$ magnitudes between 23.5 and $24.5 \mathrm{mag}$, reaching roughly one magnitude fainter than shown in Figs 9, 11, 12, and 13 (i.e. the $M_{V}<-7.8$ mag criteria is equivalent to $m_{V}=23.44 \mathrm{mag}$ for NGC 1566). The magnitude range was chosen to insure that the number of Class 1 and 2 objects combined is roughly the same as in Fig. 9, so that direct comparisons can be made.

The results for the fainter sample look similar to the brighter sample for Class 1 . However, for Classes 2 and 3 there are some important differences that should be kept in mind. First, the fainter sample has a larger scatter in measured colours. This is due to a combination of larger photometric errors, an increase in stochasticity (especially for Class 2 where individual stars are more prevalent), and a higher degree of classification errors.

In Fig. 19, the number of Class 1 sources which fall in Box 1 for RESNET and VGG are roughly the same as those in Fig. 9 for RESNET and VGG (i.e. 51 and 47 for the faint clusters compared to 46 and 45 for the brighter clusters). A human examination of objects that were classified by both RESNET and VGG as Class 1 shows that nearly all ( $\approx 90$ per cent) of these objects would also be classified as Class 1 in PHANGS-HST, with only three of the objects classified in the pairs and triplets bins instead. If the criterium is relaxed to include either RESNET or VGG rather than both, the number drops only slightly ( $\approx 85$ per cent). We conclude that RESNET and VGG are able to correctly identify Class 1 Box 1 objects down to $m_{V}=$ $24.5 \mathrm{mag}$, allowing us to approximately double the number of old clusters by using the deeper RESNET and VGG classifications.

A similar result is found for Class 1 sources which fall in Box 2, where we find 175 and 169 classified by RESNET and VGG, respectively, in this fainter magnitude range (see Fig. 19), compared with 157 and 148 for their brighter counterparts (Fig. 9). The comparison does not hold up as well for the faint, young Class 1 objects in Box 3, with 51 (RESNET) and 46 (VGG) for the faint objects, compared with 82 and 76 for the brighter ones in Fig. 9.

Unlike the case for the brighter Box 1 and 2, Class 1 clusters in Fig. 19 for NGC 1566, the distributions for the fainter Class 2 
and 3 clusters look quite different than their brighter counterparts in Fig. 11, with larger numbers for the older clusters in Boxes 1 and 2, and smaller numbers for the younger clusters in Boxes 3 and 4 . The most dramatic example of this difference is a decrease from 101 for the brighter clusters in Fig. 11 for VGG Box 4, to 27 for the fainter clusters in Fig. 19 in the same box. This is consistent with what we found in Fig. 6, with a low agreement fraction for faint Class 3 objects (60 per cent), and especially for faint Class 2 (40 per cent) objects. The fact that RESNET and VGG find fewer faint sources with colours consistent with young ages is the strongest systematic bias in this study.

To summarize this section, the distribution of classified objects in a $U-B$ versus $V-I$ colour-colour diagram can be used as a figureof-merit to test which of the classification methods provide the best results. We find that all four methods give comparable results for brighter sources, with the largest systematic differences being due to the presence of fewer Class 3 candidates, as designed into the PHANGS-HST selection criteria, and the smaller number of young Class 2 and 3 objects found by RESNET and VGG. An examination of the fainter objects (i.e. $m_{V}$ between 23.5 and $24.5 \mathrm{mag}$ ) show that while the Class 1 and Class $1+$ Class 2 samples are quite robust, care must be taken when using Class 2 alone or when Class 3 is used. We remind people that the treatment of Class 3 (compact associations) has been largely superseded by the multiscale approach described in $\mathrm{K}$. Larson et al. (in preparation).

\section{COMPLETENESS ESTIMATES}

Attempts to estimate completeness in cluster catalogues generally follow the approach taken by stellar catalogues, i.e. adding arteficial objects of different magnitudes to the image and then finding what fraction can be recovered. Unfortunately, the situation is more difficult for clusters, making the resulting estimates more uncertain. The fundamental problem is that in the stellar case, there is a single point spread function for all objects and these are generally just added to an image with little or no background. For clusters, the objects come in a variety of shapes (circular, elongated, wide range of asymmetries) and different sizes.

In addition, they are often embedded in variable and high background regions from the underlying galaxy, or in crowded regions since stars tend to be born in clusters and associations. All of these issues make the task of estimating completeness for clusters a difficult one.

In D. Thilker et al. (in preparation), we follow this standard approach to estimate completeness to the degree possible by adding simulated clusters to images and determining how many we recover. We also compare results from different studies (e.g. PHANGS-HST and LEGUS) to estimate relative completeness levels based on a comparison of the intersection and the union of the two studies. This typically results in an agreement in the number of objects at about the 70 per cent level down to $m_{v}=24.0$ for the various galaxies.

Below we approach the question from two different directions to provide a sanity check on aspects of completeness that might fall outside the standard practice of adding objects to an image.

\subsection{Comparisons with a human-selected catalogue}

In this subsection, we compare our classifications with a cluster catalogue that is completely manually selected (i.e. without using a candidate list to start with), in order to assess how many clusters may be missing from the normal candidate selection process described in D. Thilker et al. (in preparation), and to better understand if the different methods are identifying similar objects. A similar study was done in M83 as reported in Chandar et al. (2010a). Agreement between human-selected and hybrid methods (automatically selected followed by human classifications) was found to be $\approx 60$ per cent in that study. This is similar to the 70 per cent estimate mentioned above when comparing the intersection and the union for this study.

As an illustrative example, we perform this test in a typical field in NGC 628, as shown in Fig. 20. No candidate clusters are identified in the top panel to avoid guiding the eye. We suggest that readers electronically enlarge the image and study this field in detail, picking out objects they identify as clusters, then compare their selection with the middle and bottom panels to see if they are included in the various catalogues. The middle panel shows Class 1 (red) and Class 2 (green) clusters from the human-selected cluster classification (large circles) and the PHANGS-HST catalogues (small circles). Class 3 (blue) and Class 4 (yellow) are also included for PHANGS-HST. The bottom plot uses the same colour scheme but includes all five classifications from PHANGS-HST (smallest circles), to LEGUS, to RESNET, to VGG, to the human-selected classifications (largest circles).

The middle panel of Fig. 20 shows good general agreement between the human-selected and PHANGS-HST classifications, with 14 of 14 exact matches for Class 1 (red) and 6 of 11 exact matches for Class 2 (green). For Class 2, three of the non-matches were classified as Class 1, one was classified as Class 3 (compact association), and one was classified as Class 4 .

These numbers are similar to those found in Figs 5 and 6, and in Wei et al. (2020), although the 14 for 14 Class 1 matches is noteworthy and probably reflects the fact that the field is not as crowded as some other regions of this and other galaxies in our sample.

Next, we note that there are four (all Class 2) of 29 clusters in the human-selected catalogues with no counterpart in PHANGSHST (i.e. 86 per cent match) and conversely, five of 28 (i.e. 82 per cent match) of the PHANGS-HST (Class $1+2$ ) clusters with no counterparts in the human-selected catalogue. These numbers are higher (better) than the roughly 60 per cent number found in Chandar et al. (2010b), probably because we are working at a brighter magnitude cutoff $\left(m_{V}=23.0\right)$ and only including Class 1 and Class 2 clusters in the comparison. Hence, we conclude that the number of 'missing' Class 1 and Class 2 clusters due to different selection of the original candidates is relatively low, approximately in the 15 per cent range down to $m_{V}=23 \mathrm{mag}$.

Most of the human-selected clusters with no counterparts in Fig. 20 are near the faint end of the sample, and several are just outside the MCI polygon used for selection for the PHANGS-HST sample (see D. Thilker et al., in preparation).

Hence the mismatch is not due to radical differences in morphology (e.g. very diffuse clusters) but to normal uncertainties such as visually estimating the appropriate brightness limit for the magnitude cutoff, and estimating whether the profile of a faint object can be distinguished from a star.

In the bottom panel of Fig. 20 we show sources classified as Classes 1 and 2 from all five methods. It is reassuring to find 12 clusters with Class 1 classifications in all five. However, we also note that there are no Class 2 objects with this level of agreement. This reflects the lower agreement fractions in Figs 5 and 6 for Class 2. Following this line of inquiry we find that there are eight objects which agree in three or four out of the five classification methods. At the other end, we find 12 objects that are only identified by one method, and two objects identified by two classification methods.

A few other trends that can be established by a careful examination of Fig. 20 are: (1) for the objects with four or five classifications in common, the machine learning programs (RESNET and VGG) find 
fewer Class 2 objects than the human-selected classifications (this has already been seen in Fig. 11 and Fig. 19), (2) over 50 per cent of the artifacts in the PHANGS-HST catalogue (the small yellow circles in the middle panel) are pairs and triplets, and (3) these artefacts are generally found in the more crowded regions of the image, as might be expected.

While the conclusions we can draw based on one illustrative example are limited, overall we find the agreement fairly reassuring and supportive of the general conclusion that the machine learning classifications (RESNET and VGG) are competitive with the human classifications. In addition, we found no clear cases of large diffuse clusters that are missing in this field.

\subsection{Estimating completeness for objects brighter than $M_{V}$ of $-10$}

Another method of estimating completeness for a subset of the data is to determine how many of the objects with magnitudes brighter than that expected for the brightest star (i.e. $M_{v}=-10 \mathrm{mag}$, the Humphrey-Davidson (H-D) limit; Humphreys \& Davidson 1979) are classified as clusters. This approach has two potential issues. The first is that some of these bright objects are foreground stars. In principle, these can be identified using parallax measurements from GAIA, an approach being investigated in D. Thilker et al. (in preparation). The second potential problem is that some of the brightest, youngest clusters are likely to be ultracompact (e.g. Smith et al. 2020), and hence may be difficult to distinguish from individual stars, especially in more distant galaxies. Incompleteness due to limitations of spatial resolution is also relevant for the smaller clusters in general, and is a major component of the completeness testing being performed in D. Thilker et al., (in preparation).

We use NGC 628, the closest of our five galaxies at $9.8 \mathrm{Mpc}$, to make a first check of completeness at the bright end. Nineteen objects brighter than $M_{V}=-10$ mag are found, with four classified as saturated stars by PHANGS-HST. On inspection these are all obvious stars with diffraction spikes and Airy rings. A fifth object was classified as the nucleus of NGC 628 (Class 4.6). Three additional sources were classified as stars (Class 4.1). Of these, two are clearly stars since they show Airy rings, while the third is potentially an ultracompact cluster, with FWHM $=2.2$ pix. However, it remains possible that this source is a foreground star, and GAIA (DR2) measurements are inconclusive because they have a large uncertainty. One object classified as Class 4.10 (= too faint to tell) is likely to be a cluster based on its location in an intense star-forming region, even though its visual appearance and FWHM do not clearly distinguish it from a point source. This object is the only clear case of a bright cluster that is missing from the PHANGS-HST NGC 628 catalogue. Hence, 10 of 11 objects (or 10 out of 12 if the potential ultracompact cluster is included) appear to have been correctly classified as clusters. The completeness for the brightest Class 1 and Class 2 clusters therefore appears to be in the 80 to 90 per cent range for NGC 628, based on the PHANGS-HST catalogue.

The classification of H-D objects is slightly worse when the LEGUS, RESNET, and VGG classifiers are used, with eight, seven, and seven of the 10 clusters, respectively, identified as Class 1 or 2 objects. The most common misidentification are Class 4 sources (i.e. objects interpreted as a single star) as might be expected. The object identified as the nucleus by PHANGS-HST (i.e. Class 4.6), is classified as Class 4 by LEGUS, but as Class 1 by both RESNET and VGG. Hence, researchers should be careful to check the classification of the brightest objects if their study is sensitive to them. All four methods classified the foreground stars correctly.

\subsection{Issues related to completeness and systematic differences in} classification

\subsubsection{Double counting}

The DOLPHOT package used in PHANGS-HST for both stellar and cluster detection was designed for crowded stellar fields. It uses an iterative approach, finding peaks, fitting the PSF and subtracting it, and then refitting to see if new peaks can be detected. While this works well if all the objects are stellar, in fields where both stars and clusters are present the software sometimes detects additional, false peaks after it subtracts out slightly resolved clusters.

These false peaks are removed during the human classifications and designated Class 4.9 (= redundant - see Table 3). Operationally this is performed by classifying the brighter object and then giving any candidate within a radius of five pixels a value of Class 4.9. This procedure also avoids double (or more) counting in Class 3 compact associations. LEGUS does not include this redundancy check, hence the much larger number of redundant (multiply detected) Class 3 objects in Fig. 17.

If uncorrected, the number of multiple detections of Class 3 objects for the RESNET and VGG classifications would be even higher than for LEGUS. For this reason, redundant objects are removed from the RESNET and VGG machine learning classifications in a post-processing step of the pipeline (see D. Thilker et al., in preparation) using the same algorithm as employed for the human check. This results in numbers which are roughly 50 lower than for LEGUS in Fig. 12. The numbers are still considerably higher than for PHANG-HST due to the removal of pairs and triplets as artifacts (i.e. Classes 4.2 and 4.3), as discussed in Section 5.

\subsubsection{Trends in classification based on confusion matrices}

A standard tool used in many machine learning studies is the 'confusion matrix'. ${ }^{3}$ This graphic provides a concise way to visualize how often the classifications are in agreement, as well as insight into the most common types of systematic differences.

Fig. 21 shows the confusion matrices for the six comparisons between different methods for the bright $\left(m_{V}<23.5 \mathrm{mag}\right)$ objects in NGC 1566. Ideally, the darkest colour (highest percentage of matches) would be found along the diagonal. In all six cases we find the highest matching fraction is for Class 1 objects (approximately 80 per cent), as we also did in Fig. 5 and Table 2.

The off-diagonal values allow us to see where the most common systematic differences in classifications occur. For example, we note that a common difference is for Class 4 objects from PHANGS-HST to be classified as Class 2 by LEGUS (i.e. 29 per cent of the time according to the top left-hand panel in Fig. 21). This is primarily due to the difference in how pairs and triplets are classified by the two studies, as shown in Fig. 12 and discussed in Sections 3.2 and 5. The converse of this (i.e. a PHANGS-HST Class 2 object being classified as a LEGUS Class 4 object) is very rare, only 5 per cent of the time according to the upper left-hand matrix in Fig. 21. This is the largest systematic difference between the two studies.

Is the same trend seen between LEGUS and the two machine learning methods? Fig. 21 shows a similar strong trend does exist

\footnotetext{
${ }^{3}$ Our procedure for making confusion matrices is non-standard, due to the fact that we do not define one study as 'ground truth' but instead average the results for the two studies being compared, as discussed in Section 4. This works fine for the diagonal, but for the off-diagonal values it requires a reflection across the diagonal to identify the appropriate box to average.
} 


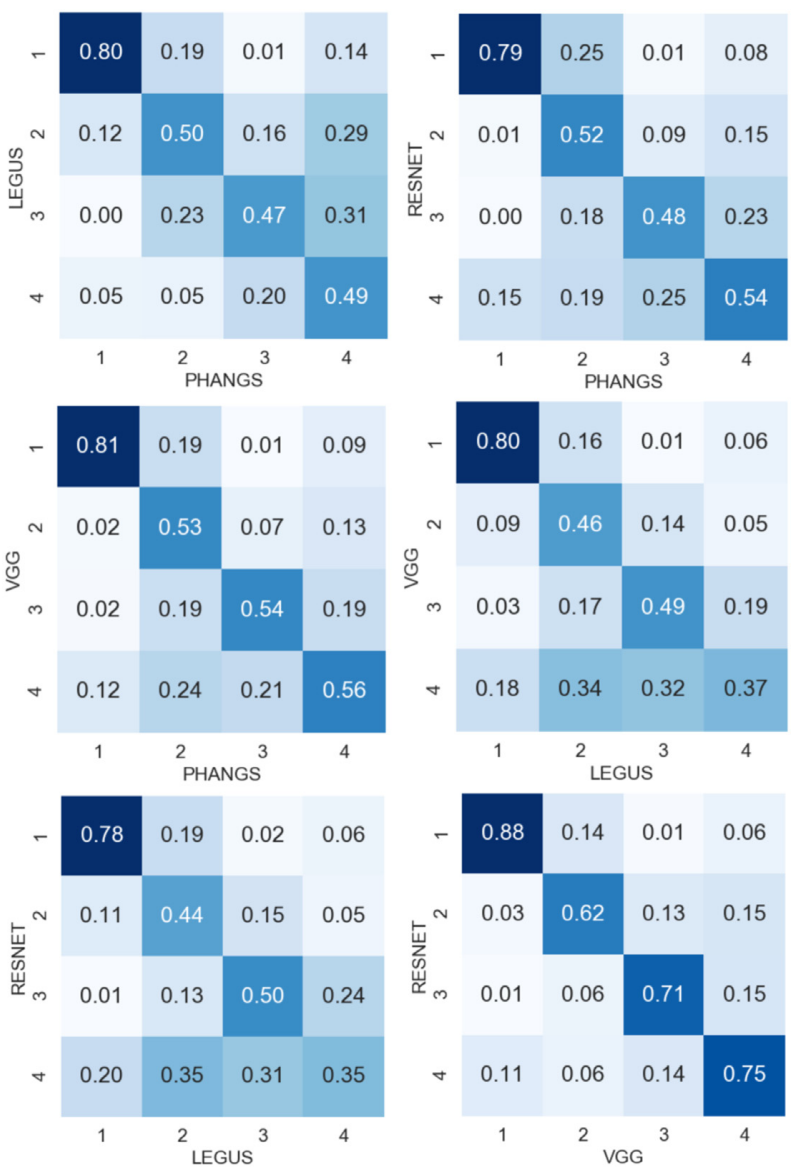

Figure 21. The confusion matrices for all six comparisons between PHANGS-HST, LEGUS, RESNET, and VGG for NGC 1566.

for LEGUS to classify objects as Class 2 that RESNET and VGG classify as Class 4 , (i.e. 35 per cent and 34 per cent values versus 5 per cent and 5 per cent) in the two panels with LEGUS on the Xaxis. Hence this tendency is not unique to the PHANGS-HST versus LEGUS comparison.

Also of interest are cases where both an off-diagonal value and its converse are high, indicating a large random uncertainty (i.e. hard to classify) rather than a systematic difference. The best example is that all the comparisons between Class 3 and Class 4 are between 14 per cent and 32 per cent, even for RESNET versus VGG.

On the other end of the scale, Class 1 objects are almost never mistaken for Class 3 objects in any of the comparisons (i.e. between 0 and 3 per cent in all 12 relevant comparisons in Fig. 21).

An interesting comparison involving the machine learning classifications is a strong tendency for both RESNET and VGG to classify objects as Class 1 that PHANGS-HST classifies as Class 2 (i.e. 19 per cent and 25 per cent of the time. The converse is only found 1 per cent and 2 per cent of the time. This same effect was found in Fig. 20. A comparison between the two machine learning algorithms alone (bottom-right-hand panel) shows that RESNET has a stronger tendency to find Class 1 rather than Class 2 objects (i.e. 14 per cent compared to 3 per cent) when compared to VGG. We note that using a sample including both Class $1+2$ (i.e. the 'standard' sample) will eliminate this problem. Hence, while the largest systematic differences are caused by different definitions (e.g. whether pairs and triplets belong in Class 2 or Class 4 - differences of $\sim 25$ per cent), we also find that the two machine learning classifications can have sizeable differences (up to $\sim 10$ per cent), even though they used the same training sample.

Only NGC 1566 has been used as an illustrative example above. However, we find very similar trends in the confusion matrices for all five galaxies.

\subsubsection{Systematic differences between PHANGS-HST and LEGUS}

The primary difference between PHANGS-HST and LEGUS classifications appears to be the inclusion of more pairs by LEGUS (e.g. Sections 6.1). Here, we make a more systematic comparison by examining the number of objects classified as pairs by PHANGSHST but identified as Classes 1, 2, or 3 by LEGUS, RESNET or VGG in NGC 1566. For LEGUS classifications of PHANGS-HST pairs, we find 18 of 478 (4 per cent) identified as Class 1, 43 of 404 (11 per cent) identified as Class 2, and 32 of 691 ( 5 per cent) identified as Class 3 objects. The percentages are lower for RESNET ( 3 per cent, 5 per cent and 3 per cent) and for VGG ( 3 per cent, 4 per cent, 2 per cent). While the percentages of these systematic differences in identifications arising from close pairs of stars are relatively small, we note that the most affected objects are once again Class 2 (asymmetric clusters).

Differences in how studies define a cluster or association are relevant to the discussion of completeness. A specific borderline class that might be considered are triplets. In PHANGS-HST these are considered Class 4 artifacts (as shown in Table 3), while LEGUS generally includes triplets in Class 3 . From the numbers in Table 3 we see that including or excluding triplets changes the total number of clusters and compact associations by about 15 per cent. Hence, while this is an important contribution to the overall estimate of completeness, it is not a dominant component. One of the reasons we have classified the triplets separately is to allow users to include them in their definition of clusters and associations, if they choose.

To summarize this section, the fact that clusters come in many shapes and sizes, and that different studies use somewhat different definitions for clusters and associations, makes it challenging to estimate completeness. Completeness can be as high as 90 per cent or better for Class $1+2$ clusters brighter than $m_{v}=23.5$ mag found in uncrowded regions or those with low background (e.g. Section 6.1), and as low as 10 per cent for Class 3 sources when compared with stellar associations defined using a watershed approach (K. Larson et al., in preparation). Various sanity checks performed in this section suggest typical completeness numbers in the 70 per cent to 80 per cent range when considering Class $1+2$ clusters over the full ensemble of environments. A more detailed look at the question of completeness as a function of magnitude, crowding, background, and other properties will be included in a future study (D. Thilker et al., in preparation).

\section{DEPENDENCE OF SCIENCE RESULTS ON CLUSTER CLASSIFICATION METHOD}

How much do science results, such as the shape of the cluster mass and age distributions, depend on the classes and classification algorithm used? Some studies (e.g. Bastian et al. 2012; Krumholz et al. 2019; Adamo et al. 2020) have concluded that source selection is the major factor which has led different groups to reach different conclusions. Other studies (e.g. Chandar et al. 2014) find that the mass and age functions are, within the errors, similar when catalogues using different selection criteria are used.

In this section, we address the question using the four main classification methods for a single galaxy, NGC 1566. A. Mok 

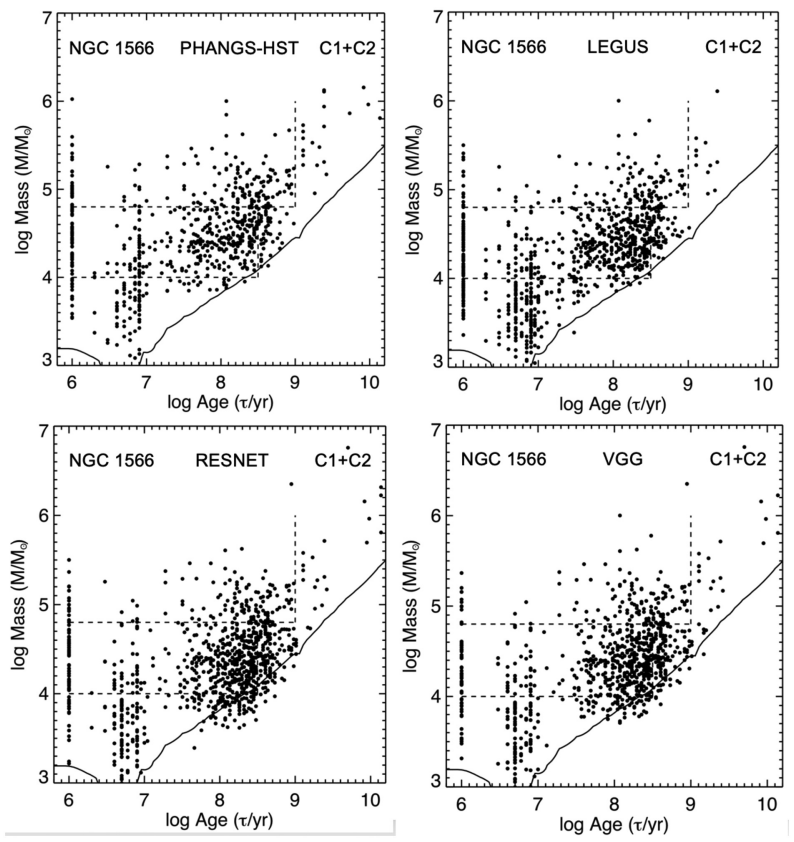

Figure 22. Log mass versus log age diagrams for NGC 1566 for the four classification methods using the Class $1+2$ classifications. The dotted lines show the various cuts used in the determination of mass and age functions.

et al. (in preparation) will examine a larger sample of PHANGSHST galaxies in the future. Fig. 22 shows the mass-age diagrams of Class $1+2$ clusters in NGC 1566 separately based on all four classification methods. Ages are taken from the non-stochastic $\chi^{2}$ SED fitting rather than the Bayesian analysis (see Turner et al. 2021). The mass-age distributions look similar, supporting the idea that the machine learning classifications provide results similar to those based on human classifications. There are fewer clusters in the PHANGS$H S T$ and LEGUS samples since they have brighter magnitude cutoffs than the RESNET and VGG samples.

Cluster mass functions can be described, to first order, by a simple power law, $\mathrm{d} N / \mathrm{d} M \propto M^{\beta}$. Fig. 23 shows the Class $1+2$ cluster mass functions in the three indicated intervals of age, again for the four different classification methods. Here, the mass functions are plotted with an equal number of objects in each bin, and the power-law index $\beta$ is determined from the best linear fit. The best-fitting values of $\beta$ are compiled in Table 4. We find that the mean and standard deviation of all 12 determinations are $-1.86 \pm 0.04$. The mean values of $\beta$, found by averaging the three different age ranges together for each classification method, are $-1.80,-1.91,-1.86,-1.88$ for PHANGS-HST, LEGUS, RESNET, and VGG, respectively. All four are within the $2 \sigma$ error estimates, and are consistent with being drawn from the same distribution. Repeating the exercise using a sample that includes only the Class 1 clusters yields values of $\beta=$ $-1.90 \pm 0.03$ for the mean of all 12 estimates, and $-1.85,-1.86$, -1.95 , and -1.93 for the four methods separately. These are again all within $2 \sigma$ uncertainties, and indicates that the mass function is insensitive to the specific classification algorithm and whether or not Class $1+2$ or Class 1 clusters are used.

Fig. 24 shows the age distributions based on Class $1+2$ clusters in NGC 1566, and Fig. 25 shows the results if only the Class 1 clusters are included. The age distribution can be fit by a single power law, $\mathrm{d} N / \mathrm{d} \tau \propto \tau^{\gamma}$. In these figures, the inner (youngest) data points, shown with open symbols, are excluded from the fits because they are systematically high compared with the rest of the distributions.
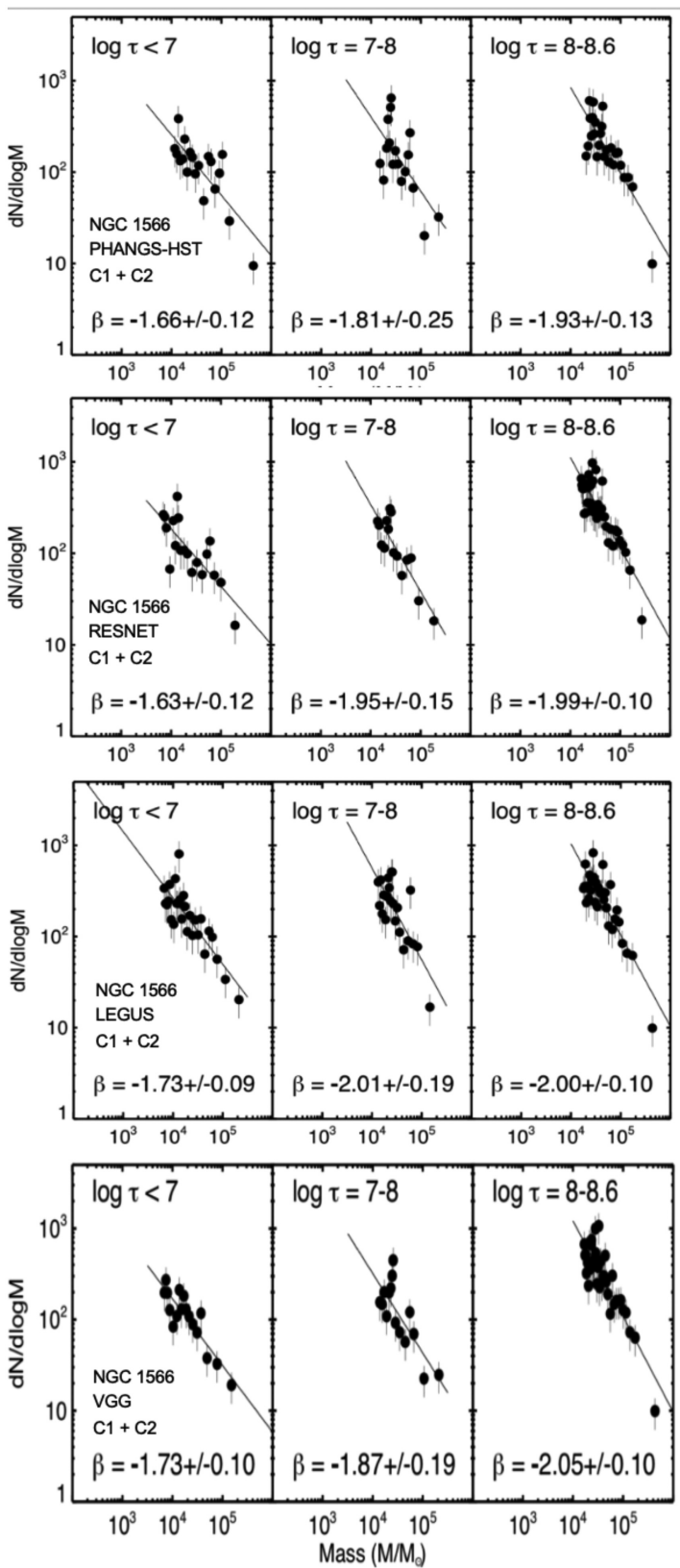

Figure 23. Mass functions for NGC 1566 for each of the four classification methods using the Class $1+2$ classifications. Fits are made for three age ranges for each method. The overall mean value is $\beta=1.86$ with an uncertainty in the mean of 0.04. The mean values for each method are the same within 2 sigma limits.

In Table 5, we present fit results for the power-law index $\gamma$ both with and without the youngest data point included in the fit.

For the Class $1+2$ sample in NGC 1566, the mean and standard deviation of all eight fits (excluding the youngest age bin) gives $\gamma=-0.45 \pm 0.03$. The mean values of $\gamma$ (averaging the two mass ranges together) are $-0.51,-0.53,-0.42,-0.34$ for PHANGS- $H S T$, LEGUS, RESNET, and VGG, respectively. We note there is tentative evidence that the machine learning classifications give slightly flatter values for $\gamma$ at about the $3 \sigma$ level. If we repeat this exercise but now include the youngest age bin, the results are: $\gamma=-0.68 \pm 0.03$ from all eight fits, and $-0.75,-0.74,-0.66$, and -0.58 . Including the data point representing the youngest age range in the fits therefore results 
Table 4. Mass function fits for NGC 1566.

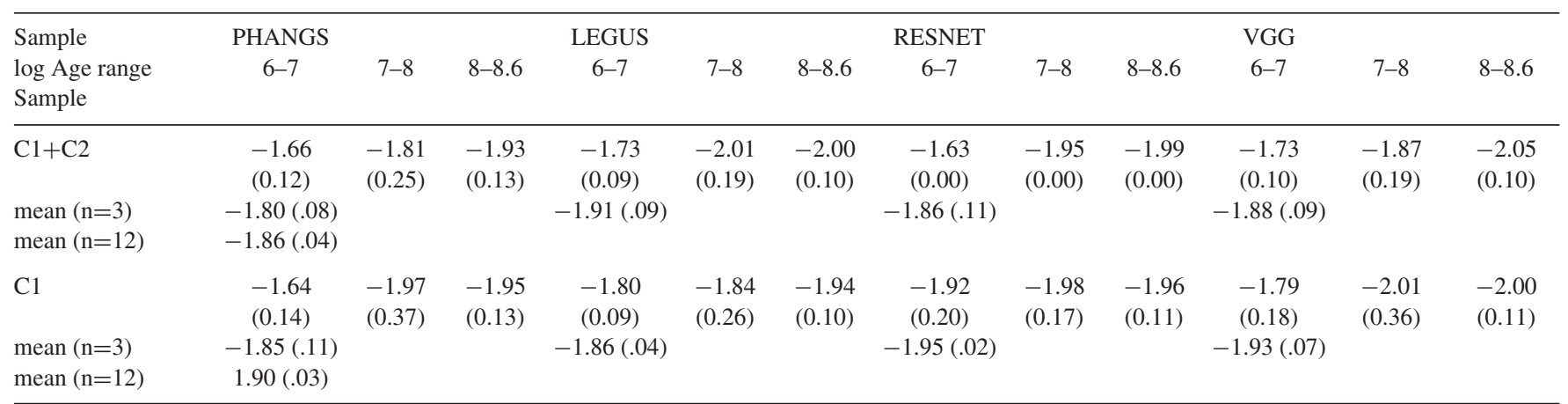
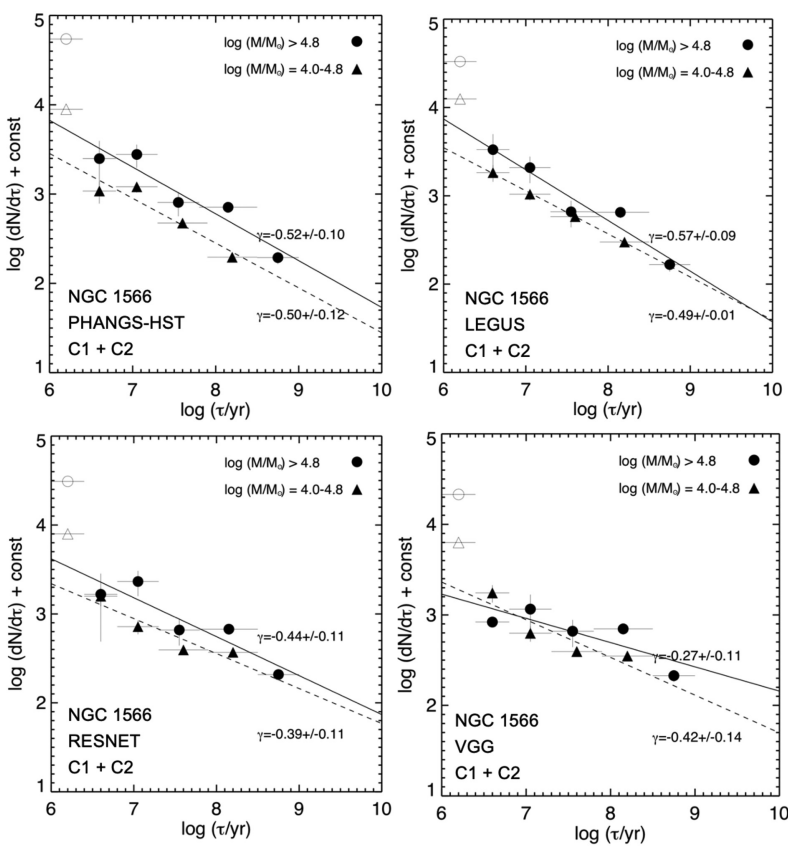

Figure 24. Age distributions for NGC 1566 for each of the four classification methods using the Class $1+2$ sample. Fits are made for two mass ranges for each method. The youngest points are shown as open symbols and are not included in the fits since they appear to be systematically high. The overall mean value is $\gamma=-0.45$ with an uncertainty in the mean of 0.03 .

in a power law index $\gamma$ for the age distribution which is steeper by $\approx-0.2$ than if the youngest point is excluded.

If we repeat this exercise for the Class 1 sample alone (i.e. Fig. 25), excluding the youngest age bin from the fits, we find the mean from all eight fits $\gamma=-0.31 \pm 0.03$, and the mean values of $\gamma$ from the different methods (averaging the two mass ranges together) are -0.43 (PHANGS-HST), -0.30 (LEGUS), -0.24 (RESNET), and -0.26 (VGG). Not surprisingly, the age distributions when only Class 1 sources are included are somewhat flatter, by $\approx 0.1-0.2$, than those which include Class $1+2$.

To summarize, the cluster mass functions are similar, with a powerlaw index of $\beta=-1.9 \pm 0.1$, regardless of the age interval or specific classification method that is used, or whether Class $1+2$ or Class 1 are included. The age distribution is more sensitive to the specific selection method, age interval for the fit, and object class. We find a range of values for the power-law index $\gamma$, with the steepest value $\gamma$ $=-0.68 \pm 0.03$ found for Class $1+2$ clusters fit over the full age range $(\log (\tau / \mathrm{yr})=6.0-9.0)$, and the shallowest $\gamma=-0.31 \pm 0.03$
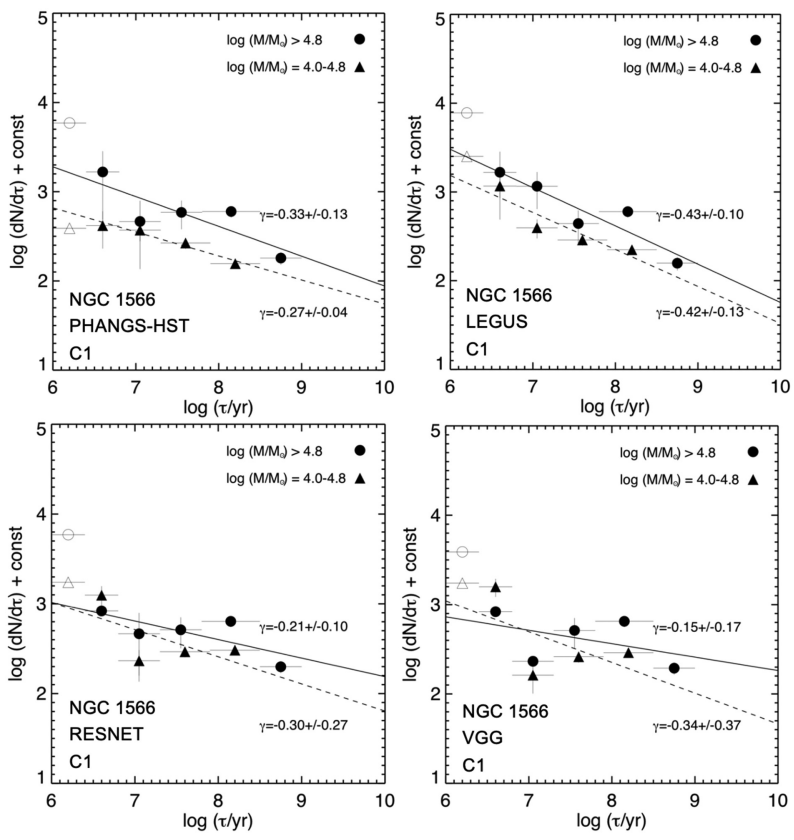

Figure 25. Age distributions for NGC 1566 for each of the four classification methods using only the Class 1 sample. Fits are made for two mass ranges for each method. The youngest points are shown as open symbols and are not included in the fits since they appear to be systematically high. The overall mean value is $\gamma=-0.31$ with an uncertainty in the mean of 0.03 .

found for Class 1 clusters where the youngest age data point is excluded $(\log (\tau / \mathrm{yr})=6.5-9.0)$. These small differences are similar to what was found in Krumholz et al. (2019). We remind the reader that these specific results are for the sample with ages taken from the non-stochastic $\chi^{2}$ SED fitting. Similar, but slightly different results are found using the Bayesian analysis (see Turner et al. 2021).

\section{FUTURE WORK}

It is reassuring that the agreement fractions for RESNET and VGG classifications based on the LEGUS-BCW training sets (see Wei et al. 2020) are roughly as good as or slightly better than those from human classifications. Based on the results presented in this work, particularly through the figures of merit, we conclude that both human and machine learning classifications do a fairly good job. In the future, we plan to continue improving the quality of the machine learning classifications so they are eventually superior to the human classifications. 
Table 5. Age distribution fits for NGC 1566.

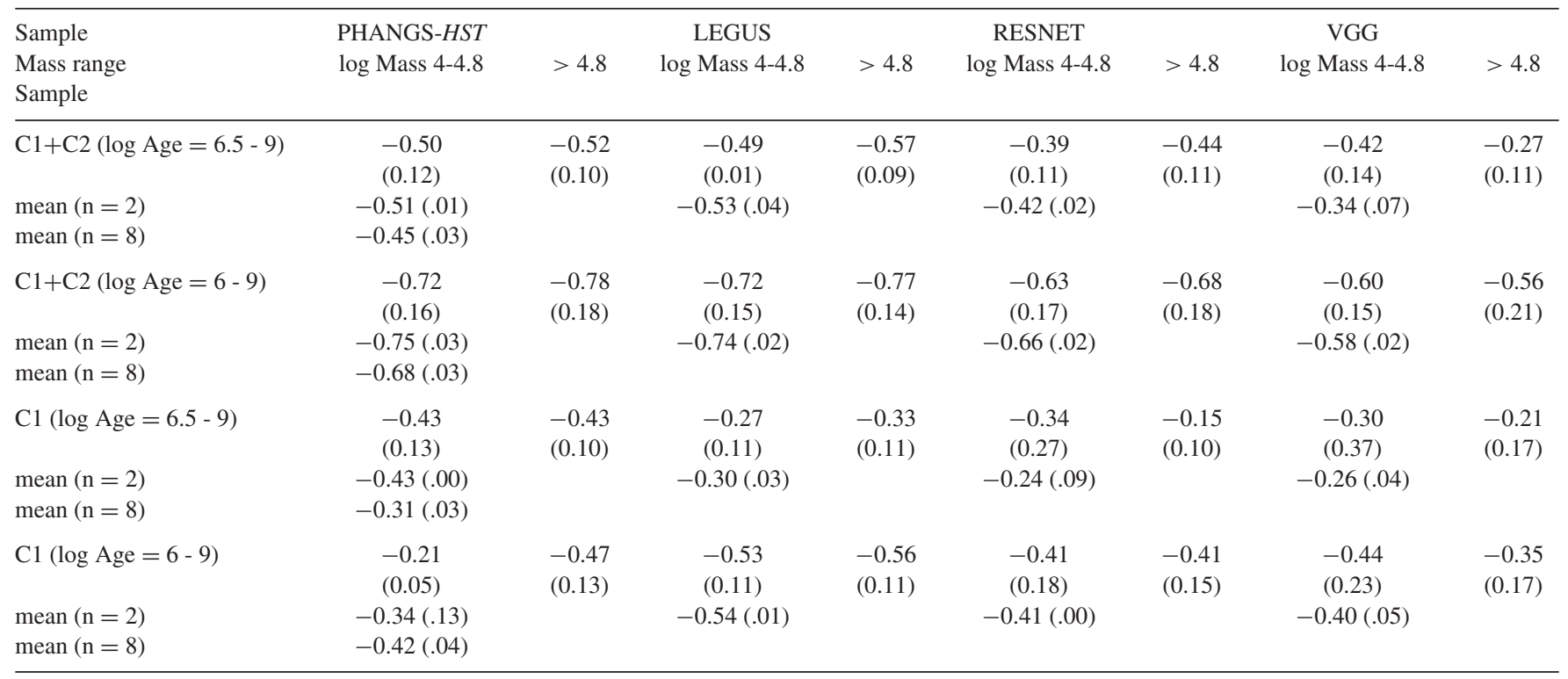

\subsection{Using different training sets}

As described in Wei et al. (2020), besides the LEGUS-BCW training sets a second training set called the LEGUS 3-person-consensus classifications was also used. The results were similar, as reported in fig. 3 of Wei et al. (2020), with the mean value of the four classes equal to 66 per cent for LEGUS-BCW galaxies and 64 per cent for the LEGUS 3-person-consensus. Due to this similarity, our first test will be to combine the two training sets to determine if the resulting classifications are improved by having larger numbers in the training set.

One trade-off to be considered is whether it is better to have a more specific (i.e. training objects taken from the same galaxy) or the largest possible training set. To test this, one of our experiments will be to use only a set of the new PHANGS-HST human classifications rather than including the previous LEGUS related training sets.

Another experiment will be to use the larger set of artifacts than just Class 4 (e.g. single dominant stars, pairs, triplets, saturated stars, diffraction spikes, cosmic rays, background galaxies, etc. see Table 3). The hypothesis is that with the wide variety of types of artefacts all mixed together, the algorithm might not be able to train as optimally as it might with similar object types in each class.

\subsection{Quality of training samples}

The quality of the training set also plays an important role in the classification accuracy of the algorithm. Some parameters that may limit the quality of the training set are crowding, magnitude, background, and distance. We plan to examine the role of each of these parameters in the future by using different subsets as training sets.

Another experiment is to identify a high quality training set by only including objects that have the same estimated classification in at least seven of the ten trials used by both the RESNET and VGG algorithms (see Wei et al. 2020 and Appendix A).

In the future, and as outlined in Wei et al. (2020), we plan to organize a cluster classification challenge using roughly a dozen different people to classify clusters in a few different galaxies. The resulting sample could be used as a training set to see if the classification agreement improves.

\subsection{Objective approaches - simulated and colour-colour selected training sets}

In this paper, we explore the development of automatically selected cluster catalogues. However, it would not be fair to call these 'objectively selected' catalogues since they are based on 'subjectively selected' training sets. In this subsection, we discuss two approaches to more objectively determined catalogues.

The first approach would be to use simulations, as described in D. Thilker et al. (in preparation) as part of our future training and testing. We are currently using these simulations to test our completeness levels and will extend this approach to see how well the RESNET and VGG algorithms recover the input classifications. A similar study is Bialopetravičius et al. (2019), who used neural network classifications based on simulations for resolved star clusters in M31.

The use of simulations has a number of pros and cons. On the positive side, it provides a better estimate of 'truth', since we know what kinds of clusters are inserted. It also provides a more rigorous method of tracking dependencies on crowding, background, luminosities, and distance. On the negative side, the simulations do not capture the full range of morphologies, especially for Class 2 clusters that are by definition asymmetric and irregular.

A related approach would be to use actual clusters scaled to different magnitudes. These objects would be drawn from within the four boxes in the colour-colour diagrams introduced in Section 5 to select the objects that go into the training sets, rather than using morphology. We would explore the resulting success fractions (i.e. how many of the objects have the colours appropriate for the box they were trained for). One potential short-coming of this approach is the effect of reddening, hence we will experiment with galaxies with both high and low overall reddening.

\section{SUMMARY AND CONCLUSIONS}

Our goal in this project is to develop automated and repeatable classification methods for making star cluster catalogues that are at least as good as, and in the future hopefully better than, human classifications. Detailed comparisons have been made between catalogues of star clusters developed using human classifiers (i.e. 
PHANGS-HST and LEGUS) and convolutional neural network models (RESNET and VGG) trained using deep transfer learning techniques, as described in Wei et al. (2020). In the current paper we focus on the results for five PHANGS-HST (and LEGUS) galaxies (NGC 628, NGC 1433, NGC 1566, NGC 3351, NGC 3627). The primary results are outlined below.

(i) We describe in detail how human classifications are made for PHANGS-HST. This includes the automated identification of cluster candidates using the MCI approach described in D. Thilker et al. (in preparation), human inspection of both the $V$-band image and a colour image, examination of surface brightness profiles and measurement of the FWHM, examination of contours, and stretching of the contrast to see if the object grows like a star or a cluster.

(ii) We examine agreement fractions between pairs of methods for each of the classes. We find that results produced by the convolutional neural network models (RESNET and VGG), using models trained with the $\mathrm{BCW}$-only classifications as described in Wei et al. (2020), are comparable in quality to the PHANGSHST and LEGUS human classifications with typical agreement for the four methods around 70 to 80 per cent for Class 1 clusters (symmetric, centrally concentrated), 40 to 70 per cent for Class 2 clusters (asymmetric, centrally concentrated), 50 to 70 per cent for Class 3 (compact associations), and 50 to 80 per cent for Class 4 (artefacts such as stars and pairs of stars). Our focus is on the Class 1 and 2 clusters; work on the Class 3 associations has been largely superseded by the watershed approach developed in K. Larson et al. (in preparation).

(iii) The dependence of agreement fractions on several properties, including magnitudes, crowding, surface brightness background, and distance are explored. While the dependence on magnitude for Class 1 clusters is quite flat, the dependence of Class 2 is much steeper, with only 30 per cent agreement found at magnitudes fainter than $m_{V}=24$ mag. Using a sample of Class $1+2$ clusters together alleviates much of this problem, resulting in agreement fractions essentially the same as Class 1 alone. Crowding results in the strongest effect, showing relatively steep dependencies for all four classes.

(iv) The distribution of data points in colour-colour diagrams is used as a 'figure of merit' to test the absolute performances of the different methods, supporting the finding that the automated classifications are comparable in quality to human classifications for $M_{V}$ brighter than $-7.8 \mathrm{mag}$. The agreement is somewhat poorer for fainter magnitudes, especially for Class 2 and 3 where the machine learning methods find more old clusters, but fewer young objects than the human classifications.

(v) Issues related to completeness are explored including comparisons with a completely human-selected catalogue, identification of common trends in classification based on confusion matrices, and an examination of whether objects too bright to be individual stars (i.e. the Humphrey-Davidson limit) are being found and included in the cluster catalogues. The most important common trend is related to differences in definitions, with LEGUS including more pairs and triplets as Class 3 (compact associations) while PHANGS$H S T$ classifies them as artefacts (Class 4).

(vi) Mass and age distributions are examined for NGC 1566 as a function of the four classification methods. We find essentially the same mass functions in all four cases, and relatively weak dependencies on the classification methods for the age distributions. Using the Class 1 catalogue alone and dropping the youngest data point results in a slope in the age distribution which is a slightly shallower than using the Class $1+2$ sample or including the youngest data point.

Based on these results, we conclude that the human classifications and the machine learning classifications are of comparable quality, although there are various caveats to be aware of, as discussed in the text. We recommend that researchers experiment with both the human and machine learning cluster catalogues, and use this experimentation to provide a measure of how it affects their science results, if at all. Similarly, we recommend that researchers experiment with samples using different combinations of classes, as in Section 7 , although the Class $1+2$ catalogue can be considered a standard in many cases.

Finally, we include an appendix which provides an overview on how to apply the Wei et al. (2020) neural network models to classify star cluster candidates in other galaxies. If comparable data sets to the PHANGS-HST or LEGUS data sets are available (i.e. five-band HST observations including the F555W band for galaxies within a similar distance range), the Wei et al. (2020) models can be used. If not, researchers can develop training sets from their own data and train the algorithms themselves. We have also discussed future work to improve the models, including re-training the RESNET and VGG neural networks with our new BCW human classifications for PHANGS-HST star clusters. We will make available all of our neural network models at the same website as discussed in Appendix A, along with Jupyter notebooks that illustrate their application.

\section{ACKNOWLEDGEMENTS}

We thank the referee for a number of insightful comments that we feel have greatly improved the paper. This study is based on observations made with the NASA/ESA Hubble Space Telescope, obtained from the data archive at the Space Telescope Science Institute. STScI is operated by the Association of Universities for Research in Astronomy, Inc. under NASA contract NAS5-26555. Support for Program number 15654 was provided through a grant from the STScI under NASA contract NAS5-26555. JMDK and MC gratefully acknowledge funding from the Deutsche Forschungsgemeinschaft (DFG, German Research Foundation) through an Emmy Noether Research Group (grant number KR4801/1-1) and the DFG Sachbeihilfe (grant number KR4801/2-1). JMDK gratefully acknowledges funding from the European Research Council (ERC) under the European Union's Horizon 2020 research and innovation programme via the ERC Starting Grant MUSTANG (grant agreement number 714907). TGW acknowledges funding from the European Research Council (ERC) under the European Unionś Horizon 2020 research and innovation programme (grant agreement No. 694343). EAH and WW gratefully acknowledge National Science Foundation (NSF) awards OAC-1931561 and OAC-1934757. FB acknowledges funding from the European Research Council (ERC) under the European Unions Horizon 2020 research and innovation programme (grant agreement No.726384/Empire).

\section{DATA AVAILABILITY}

The data underlying this article are available at the Mikulski Archive for Space Telescopes at https://archive.stsci.edu/hst/search_retriev e.html under proposal GO-15654. High level science products associated with HST GO-15654 are provided at https://archive.st sci.edu/hlsp/phangs-hst. 


\section{REFERENCES}

Adamo A. et al., 2017, ApJ, 841, 131

Adamo A. et al., 2020, Space Sci. Rev., 216, 69

Bastian N. et al., 2012, MNRAS, 419, 2606

Bastian N. et al., 2014, MNRAS, 444, 3829

Baumgardt H., Kroupa P., 2007, MNRAS, 380, 1589

Bialopetravičius J., Narbutis D., Vansevičius V., 2019, A\&A, 621, A103

Bruzual G., Charlot S., 2003, MNRAS, 344, 1000

Calzetti D. et al., 2015, ApJ, 811, 75

Chandar R., Fall S. M., Whitmore B. C., 2010a, ApJ, 711, 1263

Chandar R. et al., 2010b, ApJ, 719, 966

Chandar R., Whitmore B. C., Calzetti D., O'Connell R., 2014, ApJ, 787, 17

Cook D. O. et al., 2019, MNRAS, 484, 4897

Deng J., Dong W., Socher R., Li L.-J., Li K., Fei-Fei L., 2009, in CVPR09. Available at: http://www.image-net.org/

Fouesneau M., Lançon A., Chandar R., Whitmore B. C., 2012, ApJ, 750, 60

Gieles M., Portegies Zwart S. F., 2011, MNRAS, 410, L6

Ginsburg A. et al., 2016, A\&A, 595, A27

Girichidis P., Federrath C., Allison R., Banerjee R., Klessen R. S., 2012, MNRAS, 420, 3264

Grasha K. et al., 2019, MNRAS, 483, 4707

Grudić M. Y., Kruijssen J. M. D., Faucher-Giguère C.-A., Hopkins P. F., Ma X., Quataert E., Boylan-Kolchin M., 2021, MNRAS, 506, 3239

Hannon S. et al., 2019, MNRAS, 490, 4648

He K., Zhang X., Ren S., Sun J., 2016, Proceedings of the IEEE conference on computer vision and pattern recognition. p. 770. Available at: https://www.cv-foundation.org/openaccess/content_cvpr_2016/ht ml/He_Deep_Residual_Learning_CVPR_2016_paper.html

Hodge P. W., 1981, Atlas of the Andromeda Galaxy. University of Washington Press, Seattle

Holtzman J. A. et al., 1992, AJ, 103, 691

Humphreys R. M., Davidson K., 1979, ApJ, 232, 409

Johnson L. C. et al., 2012, ApJ, 752, 95

Johnson L. C. et al., 2015, ApJ, 802, 127

Kruijssen J. M. D., Maschberger T., Moeckel N., Clarke C. J., Bastian N., Bonnell I. A., 2012, MNRAS, 419, 841

Krumholz M. R., McKee C. F., Bland-Hawthorn J., 2019, ARA\&A, 57, 227

Leroy A. K., et al., 2021, ApJS, 255, 19

Maíz Apellániz J., 2009, ApJ, 699, 1938

Messa M. et al., 2018, MNRAS, 473, 996

Meurer G. R., Heckman T. M., Leitherer C., Kinney A., Robert C., Garnett D. R., 1995, AJ, 110, 2665

Parker R. J., Meyer M. R., 2012, MNRAS, 427, 637

Parker R. J., Wright N. J., Goodwin S. P., Meyer M. R., 2014, MNRAS, 438, 620

Pérez G., Messa M., Calzetti D., Maji S., Jung D. E., Adamo A., Sirressi M., 2021, ApJ, 907, 100

Schweizer F., Miller B. W., Whitmore B. C., Fall S. M., 1996, AJ, 112, 1839

Silva-Villa E., Adamo A., Bastian N., Fouesneau M., Zackrisson E., 2014, MNRAS, 440, L116

Simonyan K., Zisserman A., 2014, preprint (arXiv:1409.1556)

Smith L. J., Bajaj V., Ryon J., Sabbi E., 2020, ApJ, 896, 84

Turner J. A. et al., 2021, MNRAS, 502, 1366

Ward J. L., Kruijssen J. M. D., 2018, MNRAS, 475, 5659

Ward J. L., Kruijssen J. M. D., Rix H.-W., 2020, MNRAS, 495, 663

Wei W. et al., 2020, MNRAS, 493, 3178

Whitmore B. C., Sparks W. B., Lucas R. A., Macchetto F. D., Biretta J. A., 1995, ApJ, 454, L73

Whitmore B. C., Zhang Q., Leitherer C., Fall S. M., Schweizer F., Miller B. W., 1999, AJ, 118, 1551

Whitmore B. C. et al., 2010, AJ, 140, 75

Whitmore B. C. et al., 2014, ApJ, 795, 156

Whitmore B. C. et al., 2016, AJ, 151, 134

Whitmore B. C. et al., 2020, ApJ, 889, 154

Wright N. J., 2020, New Astron. Rev., 90, 101549

\section{APPENDIX A: A GENERAL INTRODUCTION TO DEEP LEARNING SOFTWARE}

\author{
A1 Using the existing Wei et al. (2020) deep transfer learning \\ models
}

To encourage broad usage of our trained deep transfer learning models, we provide the trained networks, associated PYTHON scripts, and a step-by-step tutorial guide at https://archive.stsci.edu/hlsp/pha ngs-hst with digital object identifier doi:10.17909/t9-r08f-dq31.

Many aspects of the description are specific to our PHANGS-HST data set and cluster catalogues, but we attempt to provide sufficiently generalized discussion so that anyone with comparable data can classify their own sources. A short description of the main steps for classification and for new training (if desired) is provided below.

The process is reliant upon a few prerequisites. Our models have been trained using five band HST imaging in the F275W, F336W, F438W, F555W, and F814W filters. A cluster candidate catalogue containing source positions is required. The trained models from Wei et al. (2020) and our PYTHON scripts (available at the website noted above) must also be downloaded. In terms of computing resources, our PHANGS-HST classifications were accomplished using a GPU instance on Amazon Web Services (AWS). This is not required, and the work could be undertaken even on a personal desktop machine having the proper PYTHON packages (e.g. pytorch) installed. To facilitate the process, we provide a Jupyter Notebook.

We begin our classification procedure with the production of FITS subimages $(299 \times 299$ pixel $)$ centred on the position of each candidate, one subimage per band per source. These subimages are assembled into a single multi-extension FITS (MEF) file per candidate. As described in Wei et al. (2020), we generate a total of twenty classifications for each object using ten ResNet18- and ten VGG19-based models. This is accomplished with a wrapper script that makes the individual calls specific to each of the networks. Finally, the resulting classifications are consolidated into a single file in which we tabulate the per-candidate classification mean, median, mode, standard deviation, and a quality factor defined as the fraction of outcomes matching the mode, alongside the individual classifications for each of the ResNet18 and VGG19 architectures.

\section{A2 Creation of new models with deep transfer learning}

In the event that one does not wish to use the cluster classification models published in Wei et al. (2020), our scripts also provide a guide for creating new independently-trained deep learning models.

For training, the machine learning algorithm requires preclassified sets of images with the same format as described above. These must be split into a training sample and a test sample to allow the model to iteratively test its accuracy.

Depending on the processor used, batch size, and the number of batches run, the training process may take a few hours to a few days to complete. Thus, it is generally advisable to train with the greater processing power of a GPU. For example, training the ResNet 18 architecture using 10000 batches with 32 objects per batch takes about $4 \mathrm{~h}$ with the p3.2xlarge GPU instance on AWS, while training the VGG19 architecture with the same parameters will take about 50 per cent longer. These training times roughly scale with the number of batches and batch size. 
Batch size, learning rate, and number of batches all influence the effectiveness of the trained model. To create a batch, a random object is first chosen, and then a random object from the training file is selected. Next, the selected image is rotated between 0 and 360 degrees (by 90 degree intervals) and also has a 50 per cent chance of being flipped. This augmentation is designed to manufacture a larger sample of objects for training and makes it very rare for the model to train on the exact same image multiple times.

Once all of the object images in a batch are collected, the training procedure performs matrix multiplication on the original, generic model (e.g. ResNet18 pre-trained on $>1$ million images from the ImageNet data base) and then compares its predicted classes (essentially a random guess to begin with) for the test objects with their ground truth classes. The degree to which the matrix multiplication changes per iteration during training is determined by the learning rate; a faster learning rate will make larger modifications, which may train a model faster, but may also result in a less accurate final model. These steps are then repeated for the desired number of batches; thus 10000 batches correspond to 10000 modifications to the initial model. The user should note, however, that it is possible to over-train the model, where the model becomes overly adept at classifying the objects on which it trains rather than accurately classifying a completely new set of objects.

All the scripts necessary to do what is outlined in this appendix, a fully commented Jupyter notebook, and other more detailed documentation are available at the website listed above.

This paper has been typeset from a $\mathrm{T}_{\mathrm{E}} \mathrm{X} / \mathrm{LT} \mathrm{T} \mathrm{X}$ file prepared by the author. 